\title{
Cas9-induced large deletions and small indels are controlled in a convergent fashion
}

\author{
Michael Kosicki $^{1, \bigotimes}$, Felicity Allen $^{2}$, and Allan Bradley ${ }^{3, \bigotimes}$ \\ ${ }^{1}$ Lawrence Berkeley National Lab, Berkeley, US \\ ${ }^{2}$ Wellcome Sanger Institute, Hinxton, UK \\ ${ }^{3}$ Department of Medicine, University of Cambridge, Cambridge, UK
}

\begin{abstract}
Repair of Cas9-induced double-stranded breaks results primarily in formation of small indels, but can also cause potentially harmful large deletions. While mechanisms leading to the creation of small indels are relatively well understood, very little is known about the origins of large deletions. Using a novel library of clonal mouse embryonic stem cells bona fide deficient for 32 DNA repair genes, we have shown that large deletion frequency increases in cells impaired for non-homologous end joining and decreases in cells deficient for the central resection gene $\mathbf{N b n}$ and the microhomology-mediated end joining gene Polq. Across deficient clones, increase in large deletion frequency was closely correlated with the increase in the extent of microhomology and the size of small indels, implying a continuity of repair processes across different genomic scales. Furthermore, by targeting diverse genomic sites, we identified examples of repair processes that were highly locus-specific, discovering a novel role for exonuclease Trex1. Finally, we present evidence that indel sizes increase with the overall efficiency of Cas9 mutagenesis. These findings may have impact on both basic research and clinical use of CRISPR-Cas9, in particular in conjunction with repair pathway modulation.
\end{abstract}

Correspondence: mkosicki@lbl.gov, ab2592@medschl.cam.ac.uk

\section{Introduction}

The goal of genome engineering is the introduction of particular genotype (or genotypes) to the exclusion of others. A programmable nuclease Cas9 is currently the primary tool of genome engineering in clinical and basic research context. Resolution of the double-stranded break (DSB) induced by Cas9 at a location determined by a guide RNA (gRNA) is the principal cause of Cas9 mutagenesis and templated editing. Since the specific outcome depends primarily on the relative activity of different DNA repair pathways, understanding of their function in genome engineering is crucial.

Cas9 mutagenesis is primarily the result of non-homologous end joining (NHEJ) and microhomology-mediate end joining (MMEJ) repair. NHEJ is initiated by Ku70/Ku80 complex binding to the ends of the break, protecting it from degradation. A cascade of events involving, among others, DNA-PKcs, 53BP1, Xlf, Xrcc4 and Lig4, leads to either a perfect repair or a small indel $(<10 \mathrm{bp})$. Resection of the break by the Mre11-Rad50-Nbs1 (MRN) complex, promoted by Ctip and Brca1, prevents NHEJ. Resected DNA can be repaired through microhomology-mediate end joining (MMEJ), which involves Parp1, Pol凤 and ligases Lig1 and Lig3, resulting in larger indels (1).
A frequency spectrum of indels resulting from NHEJ or MMEJ repair in a population of cells, the "indel profile", is specific to local DNA sequence of the target and generally stable across tested cell lines (2-4). These indels, usually smaller than $50 \mathrm{bp}$, can be predicted from DNA sequence with high precision (5-8). In particular, frequent occurrence of $1 \mathrm{bp}$ insertions templated from around the cut site has been linked to Cas9-induced DSBs with 1 nt 5 ' overhang $(9,10)$. The size of indels is typically increased by NHEJ inhibition, while inhibition of the core MMEJ proteins, such as Parp1 and Pol $\vartheta$, decreases them $(3,11-15)$. While predictable and partially malleable, Cas 9 mutagenesis often does not lead to the desired genome engineering outcomes.

Cas9 templated editing, which hijacks the homologous recombination (HR) pathway, can lead to well-defined outcomes. If the cell is in S/G2 phase of its cell cycle and if the MRN-initiated resection proceeds further, the DSB can be repaired by HR using either sister chromatid (resulting in perfect repair) or an exogenously provided template. This process involves, among others, Brca2 and Rad51 (16). Templated repair using Cas9 is normally harder to achieve than mutagenesis and therefore a number of studies focused on increasing its frequency. In addition to optimization of transfection conditions and the template itself (e.g. 17, 18), inhibition of NHEJ proteins by pharmacological means is one of the preferred methods (e.g. 19-22). At least one company plans to test these inhibitors in the context of clinical genome engineering (23). Some of the alternative strategies include modulation of the cell cycle and of the HR pathway itself (24-28).

While a lot of literature has focused on small indels and templated repair, Cas9 complexed with a single gRNA can also induce large deletions at least kilobases in size and complex lesions, such as translocations, large insertion and noncontiguous lesions at significant frequencies (29-32). These effects were also noted in conjunction with templated editing in mice (33-36). Extensive loss-of-heterozygosity by gene conversion and megabase-long deletions were also observed (37-40). Such outcomes could be pathogenic, and may be hard to genotype. Methods which do not require DSB to introduce templated edits, such as base editing and prime editing, were developed in part to avoid such consequences. Nonetheless, these tools likely introduce DSBs occasionally, as evidenced by creation of indels, making it likely they can also introduce large deletions $(41,42)$. Furthermore, it is not 
well understood, which DNA repair pathways control their creation.

To avoid large deletions and complex lesion, we need to know which repair mechanisms lead to their creation. To study this issue, we have built a library of mouse embryonic stem cells bona fide deficient for 32 DNA repair genes, derived from a single clone constitutively expressing Cas9. Using this library, we discovered that NHEJ genes prevent large deletions, while the resection gene $\mathrm{Nbn}$ and the MMEJ gene Polq are necessary for their creation. We also find a strong correlation between the frequency of large deletions and size or microhomology usage of small indels, across a range of deficient clones. This implies that small indels and large deletions are controlled convergently. By targeting multiple genomic sites, we observed some gene deficiencies have highly locusspecific functions and discovered a new role for exonuclease Trex1. Finally, we have shown that highly efficient Cas9 editing leads to more MMEJ outcomes.

\section{Methods}

Generation of the Cas9+ embryonic stem cell clone. An EF1a-Cas9-T2A-blastR transgene in a pKLV backbone $(2,43)$ was introduced by lentiviral transduction into a highly heterozygous CB9 mouse embryonic stem cell line, derived from a cross between CAST and C57BL/6 strains (44). Low titre of the virus was used to achieve low copy number. Blasticidin selected, single cell cloned colonies were isolated and tested for Cas9 efficiency using a flow cytometric assay with self-targeting BFP-GFP-anti-GFP construct (2) or a gRNA against $C d 9$ gene (30). The most efficient and homogenous clone "CBA9" was picked for library creation.

All ES cells used in this study were propagated on SNL-blast feeder cells resistant to neomycin and blasticidin or SNLHBP feeders resistant to neomycin, blasticidin, hygromycin and puromycin. SNL-HBP were created for this purpose by stable transposition of a low passage SNL cell line with a PiggyBac PGK-hygroR-blastR-puroR construct using hyperactive PiggyBac transposase (45) and selecting a multi-resistant pool of cells. Mouse ES cells grown on SNL-HBP feeders were found to have no morphological abnormalities compared to those grown on SNL feeder cells (data not shown).

\section{Generation of the DNA damage repair deficient li-} brary. PiggyBac transposons expressing a hygromycin resistance gene and gRNAs against DNA damage repair genes (Table S1, control, knock-out and knock-out-fail gRNAs) were introduced into CBA9 Cas9+ cells in an arrayed format using lipofection. Cells were selected for stable integration using $140 \mu \mathrm{g} / \mathrm{ml}$ hygromycin and single cell cloned. gRNA-targeted loci were amplified using barcoded primers (Table S1) and sequenced using MiSeq. Mutagenic alleles were called using CRISPResso2 (46) and manual curation of reads aligned using STAR (47). The latter method yielded additional large deletion alleles ( $>50 \mathrm{bp}$ ) missed by CRISPResso2.

Based on the recovered genotype, clones were classified as "perfect", "in-frame" or "good". Clones whose all detectable alleles were frame-disrupting and, where applicable, could be assigned to a strain (one BL6 and one CAST allele), were deemed "perfect". These are very likely to have lost target gene function. Clones containing any alleles likely to be functional (frame-preserving insertion or deletions smaller than $30 \mathrm{bp}$ ) were considered "in-frame", unlikely to have lost gene function, unless a critical protein domain was affected. Other clones, including those with more than two alleles, with one allele at loci without strain specific SNPs (potentially homozygous, or harboring a complex lesion undetectable by short-range PCR), with any in-frame deletions 30 bp or larger (likely deleterious) or with alleles that could not be assigned to a strain at a heterozygous locus (because the lesion erased the distinguishing SNPs), were classified as "good". Control clones were obtained using various non-targeting gRNAs and a gRNA targeting a safe harbor Rosa26 locus. In total, 57 perfect, 18 good and 8 in-frame experimental clones, along with 12 controls, were included in the final library for a total of 95 clones. One well was intentionally left empty as a negative control for cell and DNA carry-over. The library contained clones bona fide deficient for 32 genes, with 1-4 clones for each gene (Table S2). Two of these genes, Brca2 and Xrccl, were only represented by "in-frame" clones. No clones were included for seven other targeted genes, which yielded no promising candidates (Brcal, Exol, Mrella, Rifl, Rnaseh2a, Fenl and Mad2l2).

Flow cytometric assessment of mutagenesis efficiency and complex lesion frequency. Flow cytometric assays were conducted as previously described $(30,48)$. In short, PiggyBac transposons expressing one of the five experimental gRNAs (\#15,\#33, \#48, \#48U and \#148) and a puromycin resistance gene were introduced into the library clones in an arrayed format using lipofection. Cells were selected for stable integration using $10 \mathrm{ng} / \mu \mathrm{l}$ puromycin. This strategy ensures a near complete mutagenesis. On day 7 and day 14 post-transfection, cells were stained using FLAER reagent (for PigA activity; Cedarlane) or Itga6-PE antibodies (\#313612, Biolegend) and analyzed using Cytoflex flow cytometer (Beckman-Coulter). Six replicates were performed for day 7 and four for day 14 .

Data was extracted, processed and visualized in $\mathrm{R}$, using packages flowCore, flowWorkspace, openCyto and ggcyto (49-51). The same gating was used throughout, except in replicate 6 on day 7, in which cells had to be restained and gates adjusted to lower staining efficiency. A bacterial infection was detected in replicate 1 on day 14 - cells were processed as usual and data was retained. Raw percentages of staining-positive cells from each plate (that is, replicate, experimental gRNA, staining combination) were mean and standard deviation normalized and resulting raw z-scores were decomposed using PCA. Two first principal components captured $60 \%$ of variation and separated the samples by gRNA and time of sampling. The next two components separated two batches of replicates (Fig.S1A). These batches were initiated from different master plates and used different lots of some reagents. The second batch grew faster (data not shown) and, possibly as a consequence, experienced an 
overall increased level of mutagenesis (Fig.S1B). We only used data derived from the first two principal components for analysis, expressed as a z-score with relation to mean and standard deviation of the control samples (e.g. in Fig.1B). Raw frequencies of gene expression negative cells, raw $\mathrm{z}$ scores and PCA-regressed z-scores are presented side-byside in Fig.S2.

Analysis of indel profiles. Loci targeted with five experimental gRNAs in 95 clones in two biological replicates (day 14 , replicates 5 and 6 in the flow cytometric assays) were amplified using barcoded primers (Table S1; amplicons of 244$283 \mathrm{bp}$ ) and sequenced using MiSeq. Demultiplexed reads were handled as described in Allen et al. (5). In brief, reads were transformed into indel signatures characterised by their size, type and location with respect to the cut site (3rd/4th nucleotide 5' of the PAM). For example, 'D10_L-13C2R0' is a $10 \mathrm{bp}$ deletion ('D'), whose last unmodified nucleotides are thirteen to the left of the cut site and at the cut site. Two nucleotides of microhomology ('M') could map at either end of that interval. When an indel contains insertions ('I'), microhomology indicates that this part of the insertion matches at either end of the interval, indicating a possible templated insertion.

Samples transfected using control gRNA \#33 targeting GFP were found to contain negligible amounts of indels at \#15, \#48 and \#148 sites and were discarded (data not shown). The following filters were applied to the remaining 760 samples. Indels that would result in loss of more than $150 \mathrm{bp}$ were filtered out to avoid primer-dimers ( $0.6 \%$ read loss) and samples with fewer than 400 remaining reads were removed (18/760 samples lost; each gRNA-clone combination retained at least one sample). Symmetrized Kullback-Leibler divergence ("KL") was calculated for all pairs of samples as described in Allen et al. (5). The resulting KL matrix was decomposed using multidimensional-scaling (MDS) for visualization and statistical testing. A bivariate normal distribution was fitted to controls using two first principal components of the MDS-decomposed KL matrix (Fig.S3A) and the associated p-value for each sample was calculated. An indel profile of a clone was considered significantly different from controls, if all of its replicates had a FDR-corrected pvalue $<0.01$. Analysis and visualization were performed in $R$ using ggplot2, ggrepel (https://ggrepel. slowkow . $\mathrm{com} /)$ and tidyverse group of packages, as well as colorblindr and cowplot $(52,53)$.

\section{Results}

Mouse embryonic stem cell DNA damage repair deficient library. Many DSB repair deficient cell lines have been used to study Cas9 engineering outcomes in the past. However, available lines have often been derived independently, from different mouse strains, tested using different Cas9 vectors and compared to only one or few control clones. This may introduce cryptic variability in such parameters as proliferation rate or Cas9 expression levels, which in turn may confound the effect of DNA repair deficiency. Likewise, re- cently developed pooled CRISPR drop-out screens are vulnerable to proliferation rate differences that are not related to tested phenotypes. Seeking to avoid these confounders, we built a mouse embryonic stem cell DNA damage repair deficient library, based on a single clone constitutively expressing Cas9. Mutations in 39 repair genes were introduced using Cas9 complexed with 81 gRNAs. We selected these genes to broadly cover the main DSB repair pathways, NHEJ, MMEJ and HR. We also included a number of exonucleases, expecting some of them might have a role in generating large deletions.

Approximately 800 clones (incl. controls) were created in a single experiment and passaged together, minimizing differences due to handling and reagents batches. Clones with no detectable wild-type allele and no frame-preserving indels at the target site were incorporated into the library (with few exceptions, see Methods and Table S2). As expected, attempts to mutate a number of HR genes resulted in extensive lethality and an increased number of in-frame indels (data not shown, see Methods for details). For some of these genes, we have incorporated clones with large in-frame deletions (more than $10 \mathrm{bp}$ ), expecting them to be hypomorphic. In total, we have selected 83 individual clones, with mutations in 32 repair genes. The library also included 12 control clones transfected with non-targeting gRNAs or a gRNA targeting safe harbor Rosa26 locus (Table S2).

\section{Large deletions are prevented by NHEJ and promoted} by $\mathbf{N b n}$ and Polq. Large on-target deletions and complex lesions are a significant and potentially pathogenic outcome of Cas9 mutagenesis, but DNA repair pathways contributing to these outcomes are unknown. To map out these pathways, we have applied a previously developed flow cytometric assay, which detects such lesions (30), to the newly created arrayed library of clonal mouse embryonic stem cell clones bona fide deficient for DNA damage repair genes. We transfected each clone with a gRNA against the intron of the PigA gene and measured the frequency of cells that have lost PigA expression (Fig.1A). As shown before, small indels at this site do not affect PigA expression, and the cells that have lost gene expression harbor large deletions ( $>260 \mathrm{bp}$ ) overlapping the nearest exon or, much more rarely, other complex lesions which explain expression loss (translocations, non-contiguous lesions, insertions containing polyadenylation signals). We will refer to these events collectively as "large deletions". The fact that in male ES cells there is only one copy of PigA, which is located on chromosome X, makes the assay highly sensitive.

We observed a substantial increase in large deletion frequency in clones deficient for the core NHEJ-factors, in particular Xrcc4, Lig4, Xrcc5 (Ku80 protein), Xrcc6 (Ku70), Prkdc (DNA-PKcs) and Xlf (Fig.1B). Mutations in other NHEJ genes, such as Paxx, Setmar, Dclrelc (Artemis) and Poll did not substantially influence the results, consistent with a minor role they play in this pathway. Conversely, lower frequency of deletions was found in clones mutated at the $\mathrm{Nbn}$ locus (Nbs1 protein), which is involved in initial resection of DSB leading to MMEJ and HR pathways. Similarly, dele- 
A
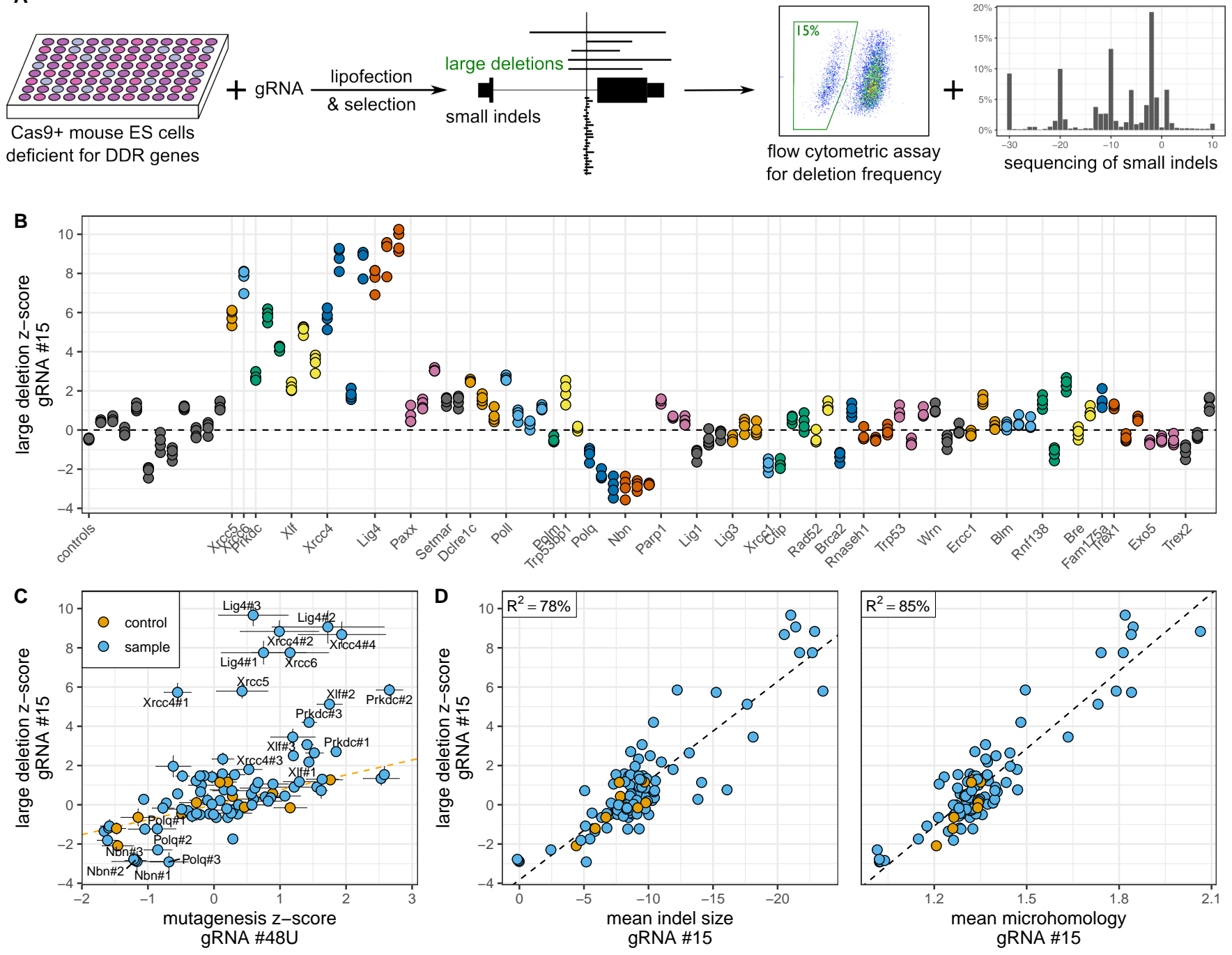

Fig. 1. End joining pathways divergently control the frequency of large deletions caused by Cas9 in mouse ES cells (A) Experimental design. Library of Cas9-positive clones bona fide deficient for DNA damage repair genes was transfected with individual gRNA-expressing constructs and selected for stable integration. Expression of target genes was measured by flow cytometry, revealing frequency of large deletions (using intronic gRNA \#15) or overall mutagenesis (using gRNAs \#48U, \#48 and \#148). Frequency of small indels was established by targeted sequencing of short-range PCR products. (B) Frequency of large deletions caused by Cas9 with intronic gRNA in DNA damage deficient clones, measured by flow cytometry, expressed as a regressed z-score (see Methods). Only the first clone in a series of clones deficient for the same gene is labeled on the $\mathrm{x}$-axis. $\mathrm{N}=4$ independent cell cultures. (C) Comparison of large deletion and mutagenesis indices (see Methods). Dashed line indicates best linear fit to control clones (in orange). Error bars are 2xSEM ( $\mathrm{N}=4$ ). (D) Correlation between large deletion z-score (measured by flow cytometry) and the size or microhomology extent of small indels (measured by targeted sequencing). Each dot represents an average readout of an individual clone ( $\mathrm{N}=1-2$ biologically independent cell cultures). Negative indel sizes indicate dominance of deletions.

tions were less common in clones deficient for Polq (Pol७), a crucial component of MMEJ pathway. Raw frequency of large deletions spanned from almost $30 \%$ in Xrcc4 and Lig4 deficient clones to around $1 \%$ in Polq and $\mathrm{Nbn}$ deficient ones, compared to $12 \%$ in control clones. Since our assay primarily detects deletions spreading in a single direction from the cut site, the true frequency of these lesions is likely 1.5-2 times higher than measured (48).

To control for the expected variability in mutagenic efficiency between individual clones, we compared the deletion frequency with results obtained using exonic gRNA \#48U, which tracks mutagenic efficiency. We chose this gRNA as reference, since mutagenesis using other exonic gRNAs was nearing saturation (\#48 and \#148; Fig.S2B, raw frequency). The effect on deletion frequency generally exceeded that on the overall mutagenesis level (Fig.1C). We conclude that large deletions are prevented by NHEJ repair and promoted by at least some part of MMEJ machinery.

\section{Small indels and large deletions are controlled by the same pathways. The effect of DSB repair pathways on} large deletion frequency was qualitatively consistent with the previously described effect of these pathways on local indel profiles. A close quantitative correlation between the two would imply a common mechanism. To see if this is the case, we sequenced a $283 \mathrm{bp}$ area around the cut site of the gRNA \#15 we have used to assess large deletion frequency. We found a strong correlation between the average size of microhomology of the sequenced small indels and large deletion frequency as measured by flow cytometry (Pearson $R^{2}=85 \%$, 
bioRxiv preprint doi: https://doi.org/10.1101/2020.08.05.216739; this version posted August 5, 2020. The copyright holder for this preprint (which was not certified by peer review) is the author/funder, who has granted bioRxiv a license to display the preprint in perpetuity. It is made available under aCC-BY-NC 4.0 International license.

A
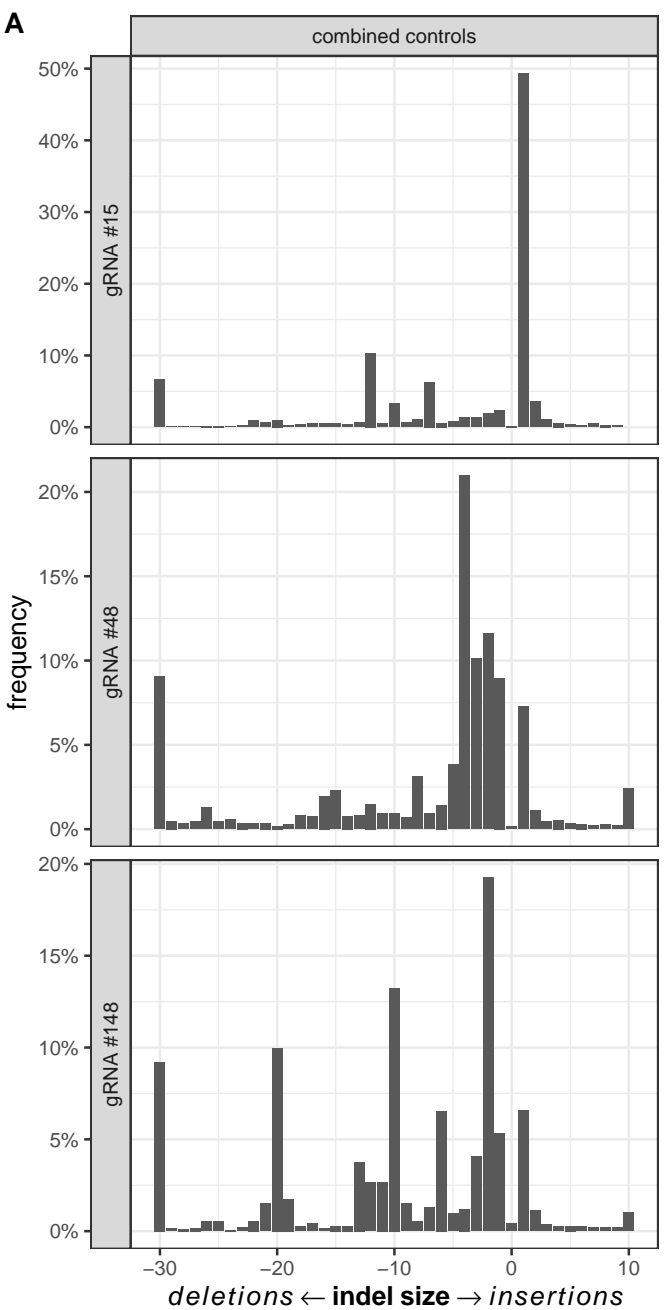

B

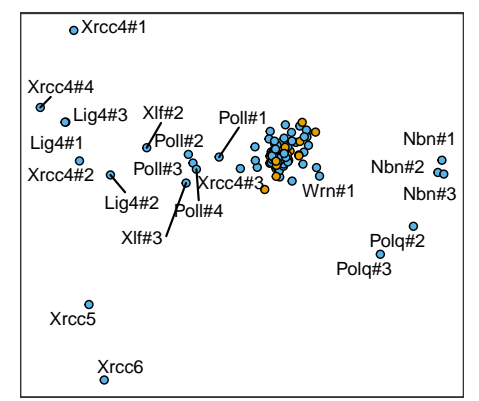

C
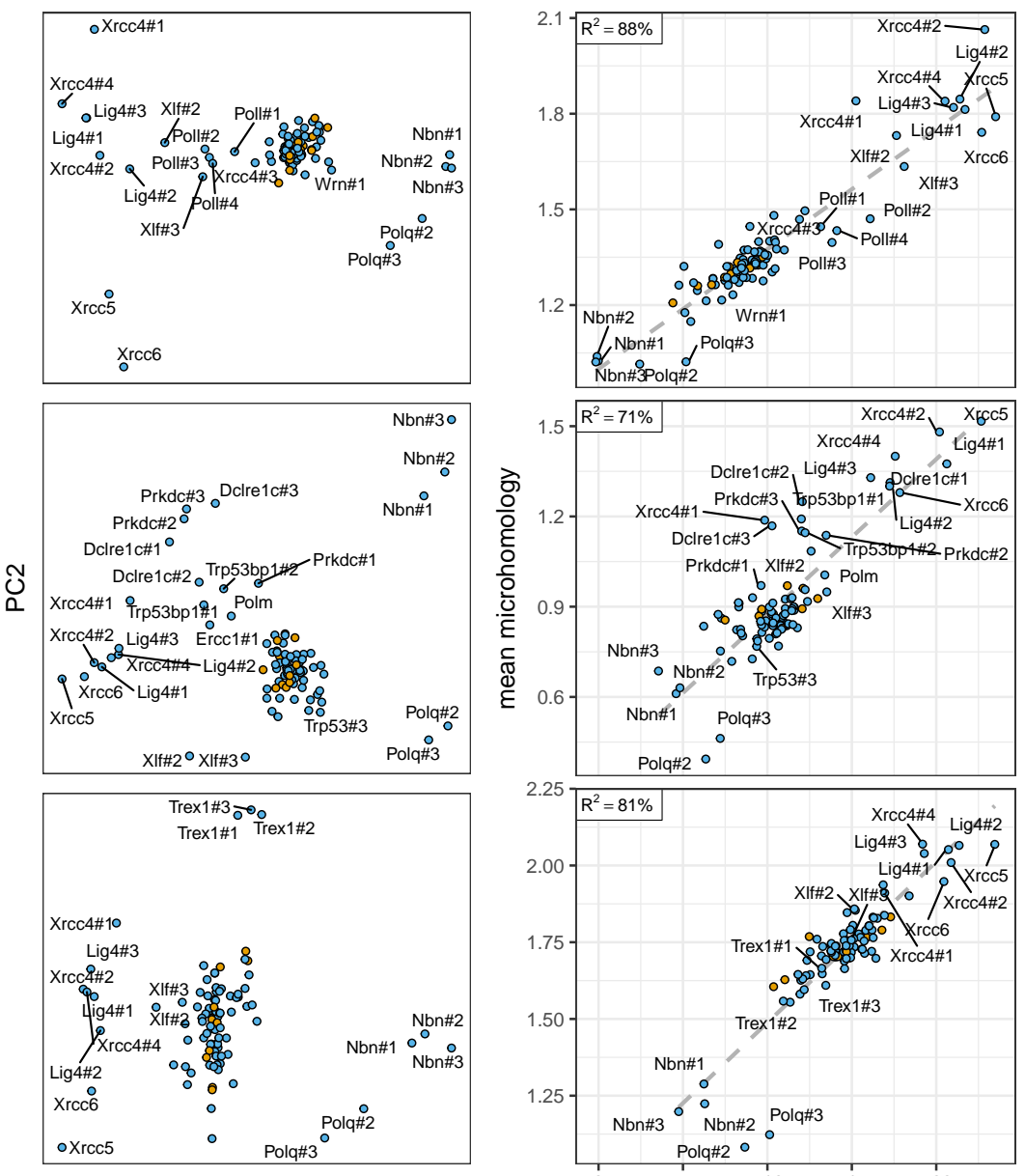

PC1

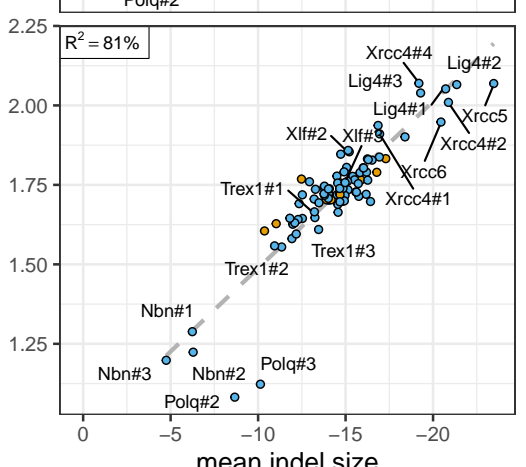

D
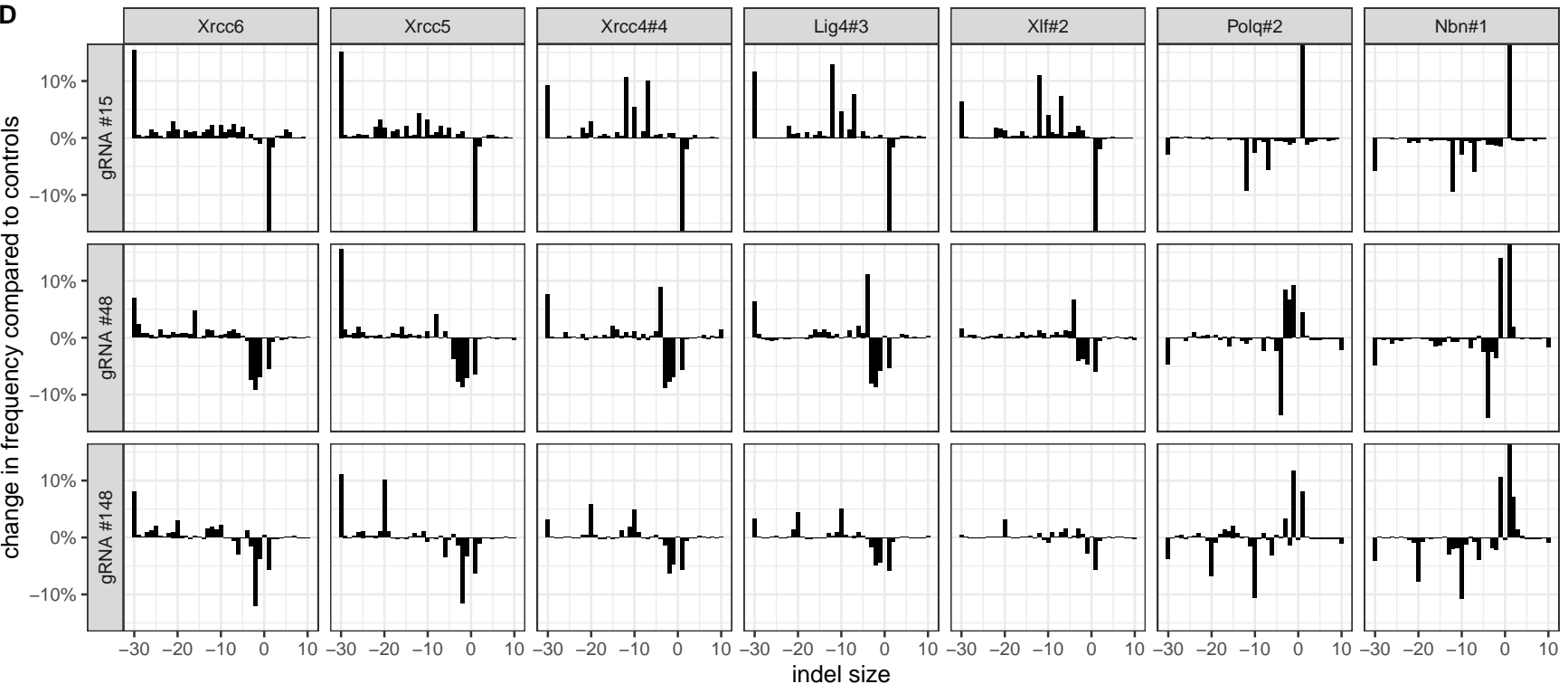

indel size

Fig. 2. Core end joining genes influence indel profiles globally. (A) Indel profiles in combined 12 control samples. (B). Relationships between cell clones based on their indel profiles. Clones significantly different from controls $(p<0.01)$ are labeled. The arrangement of non-significant clones is in Fig.S3C. (C) Correlation between mean indel size and microhomology. (D) Relative frequencies of indel sizes compared to controls in deficient clones with a significant impact on all three gRNAs. Indel profiles of other clones with significant impact are in Fig.S4. Y-axis is truncated at $-15 \%$ and $+15 \%$. In panels A and D, indel frequencies are aggregated by combined size. Negative numbers represent deletions and positive ones represent insertions. The leftmost and rightmost bars ( -30 and 10$)$ combine all larger deletions and insertions, respectively. Biological replicates $(\mathrm{N}=2)$ were averaged for clarity. All rows in panels $B$ and $C$ relate to the same gRNAs as in panel $A$. In panels $B$ and $C$, controls are in orange and samples are in blue. 
Fig.1D). We also found a moderate, inverse correlation with the average size of the small indels ( $R^{2}=78 \%$, Fig.1D). A linear model using both measures was not significantly different from single-measure models (data not shown). These observations imply a strong commonality of repair mechanisms generating both types of lesions and suggest that sequencing of short-range PCR products could be developed as an alternative assay for large deletions.

\section{Core end joining genes influence indel profiles of mul-} tiple target sites. Screens of DNA damage repair processes often rely on a single locus reporter assay or on composite readouts based on random mutagenesis. However, in vitro biochemical studies show that DNA repair is often highly sequence specific. To distinguish between universal and specific repair processes, we sequenced mutagenized target sites of three gRNAs, each with a distinct indel profile in control clones (Fig.2A). In particular, gRNA \#15 was characterized by preponderance of $1 \mathrm{bp}$ insertions, gRNA \#48 by diversity of small indels 1-5 bp in size, while gRNA \#148 induced discretely sized deletions ( $2 \mathrm{bp}, 10 \mathrm{bp}, 20 \mathrm{bp}$ ). We speculate that these profiles reflect relative contribution of NHEJ and MMEJ repair at a given site.

To obtain an overview of relationship between deficient clones, we have calculated Kullback-Leibler divergence between each pair as described in Allen et al. (5) and transformed the resulting divergence matrix using multidimensional scaling (MDS), a non-linear dimensionality reduction technique similar to principal component analysis (PCA). We found biological replicates to cluster together, indicating good reproducibility (Fig.S3A). Furthermore, the majority of clones, including all controls, clustered at the centre of the plot. This indicated most mutants did not influence the indel profile substantially, consistent with the flow cytometry assay. As a further control, we compared the frequency of mutated reads and the frequency of cells which lost expression of the target gene in the flow cytometric assay and found them to match closely for exonic gRNAs \#48 and \#148 (Fig.S3C). As expected, these numbers did not match for the intronic gRNA \#15, as in this case the two methods measure mutually exclusive outcomes: the frequency of small indels and the frequency of large deletions.

We asked which deficiencies exhibited similar effects regardless of the target site, and which other deficiencies they clustered with. Mutations in $X r c c 5$ and $X r c c 6$ genes, whose products form a functional heterodimer (Ku80-Ku70), had very similar, strong effects (Fig.2B and Fig.2D). Likewise, indel profiles of Xrcc4 and Lig4 mutants clustered together, consistent with the fact Xrcc4 forms a scaffold for Lig4. MMEJassociated Polq and Nbn clustered away from NHEJ genes such as Xrcc's 4,5 and 6 and Lig4. As shown previously, NHEJ-deficiencies increased the size of indels, while MMEJdeficiencies decreased them, although specific role of $\mathrm{Nbn}$ in indel profile modulation has not been described previously. In general, genes acting earlier in their respective pathways (Xrcc5/Xrcc6 and $\mathrm{Nbn}$ ) had stronger phenotypes than the genes acting later (Lig4, Xrcc4 and Polq).

Resection exposes single-stranded DNA, which can partic- ipate in repair using microhomology. The extent of microhomology in an indel profile could thus be confounded by the extent of resection. Taking advantage of the wide range of repair outcomes in both control and deficient clones, we decided to investigate the relationship between the two. We found a striking correlation between the average indel size (proxy for resection) and microhomology size for all gRNAs (Pearson $R^{2}$ between $71 \%$ and $88 \%$, Fig.2C). On the average, we observed $1 \mathrm{bp}$ more homology for 19-27 bp increase in indel size (depending on gRNA), with the caveat that we do not know if this relationship can be extrapolated beyond the observed intervals. We speculate that this perspective may allow assessment of the relative contribution of deficient genes to resection and microhomology repair, respectively. In particular, we think it is likely that clones close to the regression line (Trex 1, Nbn, Lig4, Xrcc5 and Xrcc6) mainly control the extent of resection, while "distal" clones (Polq, some of the significant Xrcc4 clones, Dclre1c, Prkdc and Trp53bpl) also control the extent of microhomology, at least in some genomic contexts. Consistently, Pol $\vartheta$, the gene product of the most systematically "regression line-distal" gene, is known to actively generate homologous DNA at the DSB ends. We note that clone Ercc1\#1 with gRNA \#48 was excluded from this analysis as a strong outlier, with much larger mean deletion size than controls ( $-38 \mathrm{bp}$ ), without a proportional increase in microhomology usage (1.2 bp). This may have been a consequence of insufficient read depth (1400 mutated reads) and lack of replicate sample, so this results must be treated with caution.

\section{Specialized repair pathways affect indel profiles in a locus specific manner. Having focused on indel patterns} that were common between the three gRNAs, we turned to gRNAs-specific effects. We found that Poll deficiency only had a significant effect on the profile of gRNA \#15, Trexl on \#148 and Prkdc, Dclre1c, Trp53bpl, Polm and Erccl on \#48 (Fig.2B, examples of differential indel profiles in Fig.3A). Clones Wrn\#1 and Trp53\#3 also had specific effect on gRNAs \#15 and \#48, respectively, but it was far weaker than that of other genes and did not replicate in other independently derived clones. We chose not to explore this further.

We speculated this gRNA specificity is driven by the most prominent indels in each profile. By examining individual indel frequencies we confirmed that $1 \mathrm{bp}$, microhomologyassociated insertions depleted by Poll deficiencies in profiles of all tested gRNAs, were most common in the profile of the significantly affected gRNA \#15 (Fig.3B). Analogously, microhomology-containing 7-20 bp deletions prone to Trexl depletion were the most prominent outcomes of \#148 mutagenesis. Finally, top indels depleted by Prkdc, Dclrelc, Trp53bpl, Polm and Erccl were 2-5 bp deletions, commonly induced by gRNA \#48 (Dclre1c example in Fig.3B). The effects of all deficiencies described here are consistent with the literature, except Trexl, whose function in DSB repair has not been described before.

To learn more about the effect of individual deficiencies, we examined indels that did not conform to the rules broadly laid 
bioRxiv preprint doi: https://doi.org/10.1101/2020.08.05.216739; this version posted August 5, 2020. The copyright holder for this preprint (which was not certified by peer review) is the author/funder, who has granted bioRxiv a license to display the preprint in perpetuity. It is made available under aCC-BY-NC 4.0 International license.

A

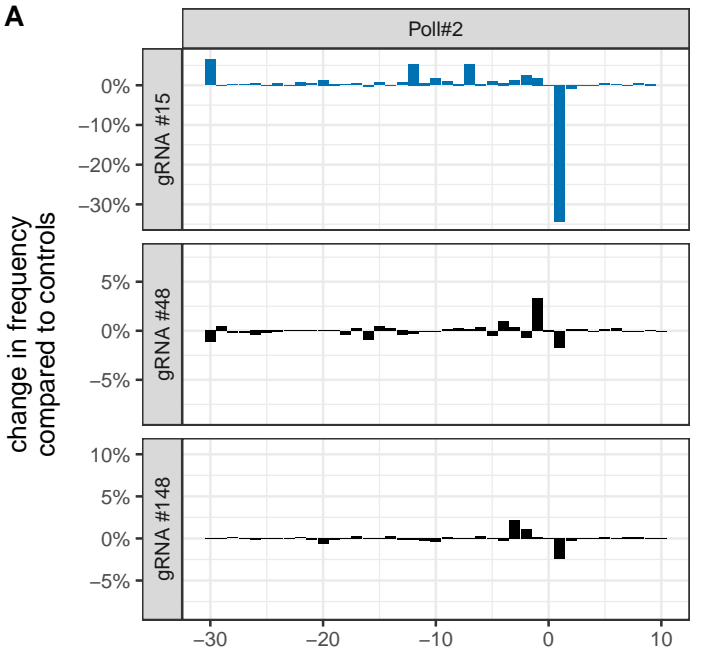

\section{B}
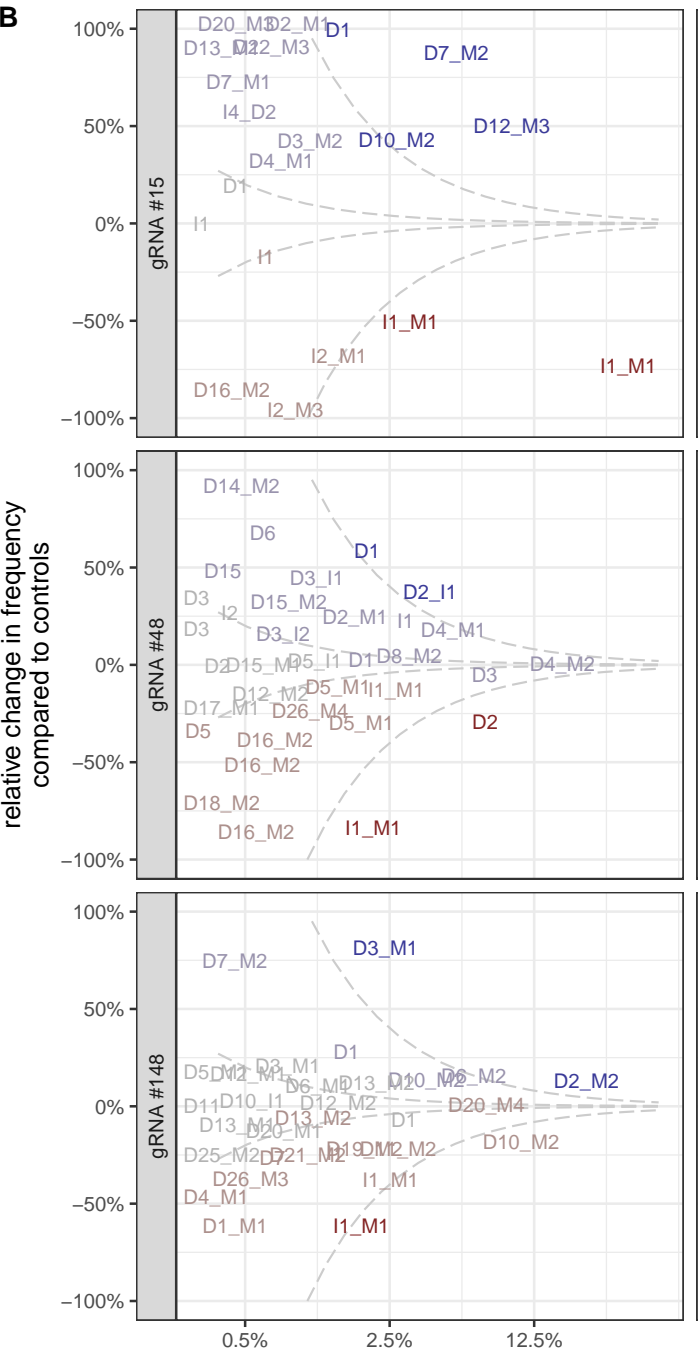
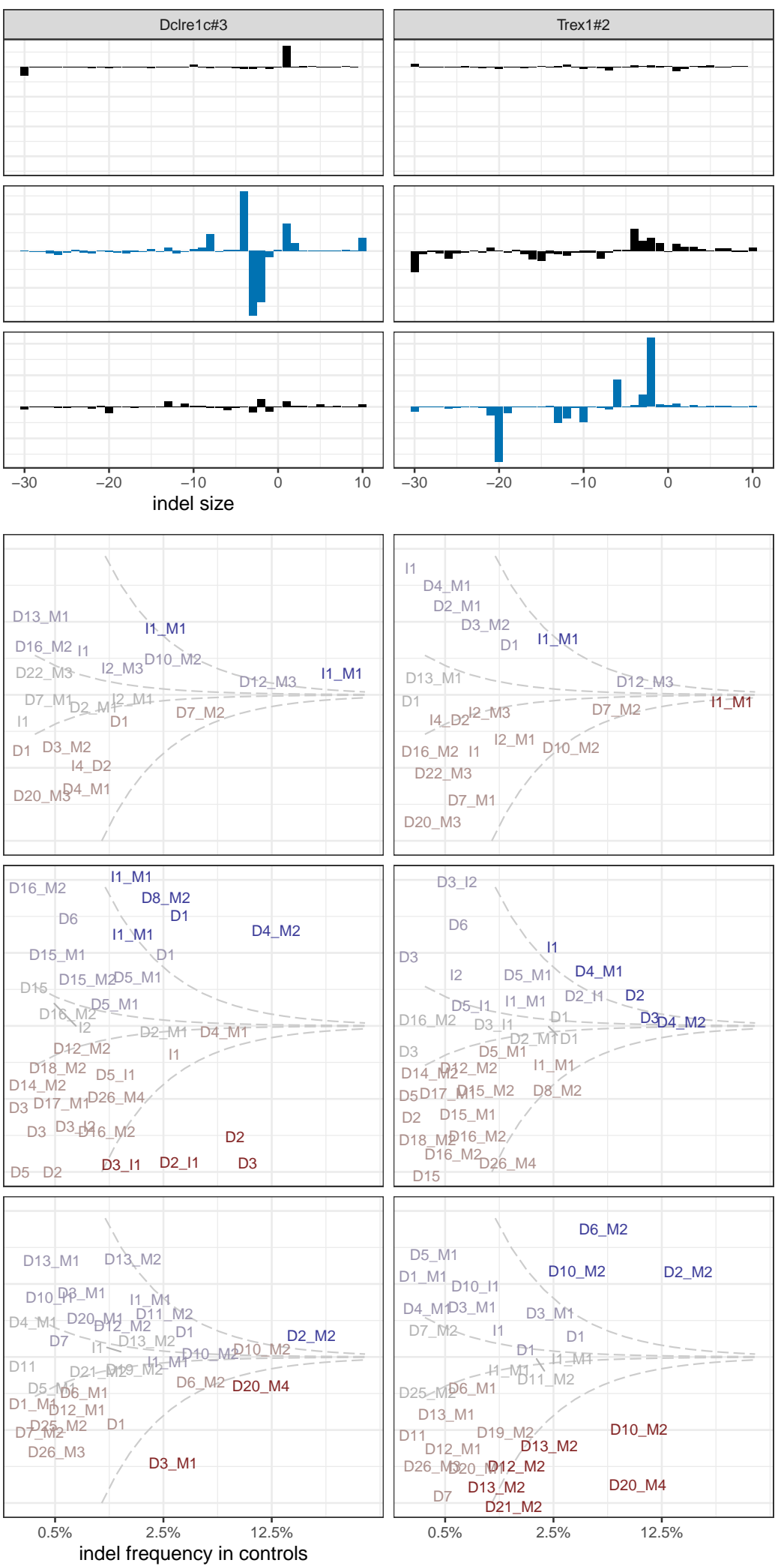

Fig. 3. Deficiencies in specialized DNA damage repair genes influence indel profiles in a locus-specific manner. (A) Indel profile divergence between controls and selected clones. Blue bars highlight clone/gRNA combinations that were significantly affected $(p<0.01)$. (B). Change in frequency of individual indels relative to controls. 'D' = deletion, ' $I$ ' = insertion, 'M' = microhomology (see Methods). X-axis indicates the frequency in control clones. Y-axis indicates relative change in indel frequency in a given clone relative to control clones. Complete loss of the indel is at $-100 \%$, while $100 \%$ indicates doubled frequency. The axis is truncated there for display clarity. Only indels present at $0.3 \%$ frequency or higher in control clones are shown. Dashed lines indicate absolute change of $0.1 \%$ and $1 \%$ respectively, color gradations highlight this change. Biological replicates $(\mathrm{N}=2)$ were averaged for clarity. All columns relate to the same gRNAs as in panel A. 
A
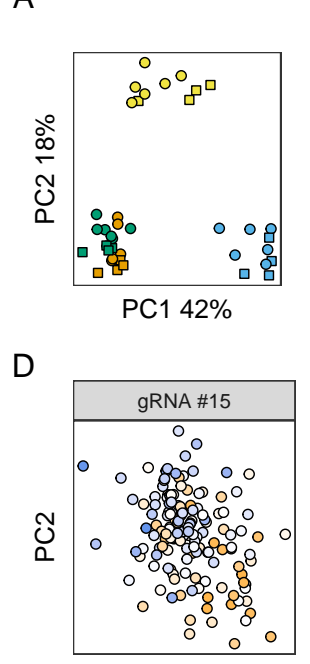

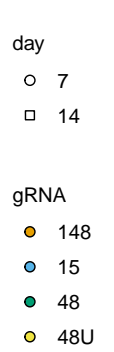

B
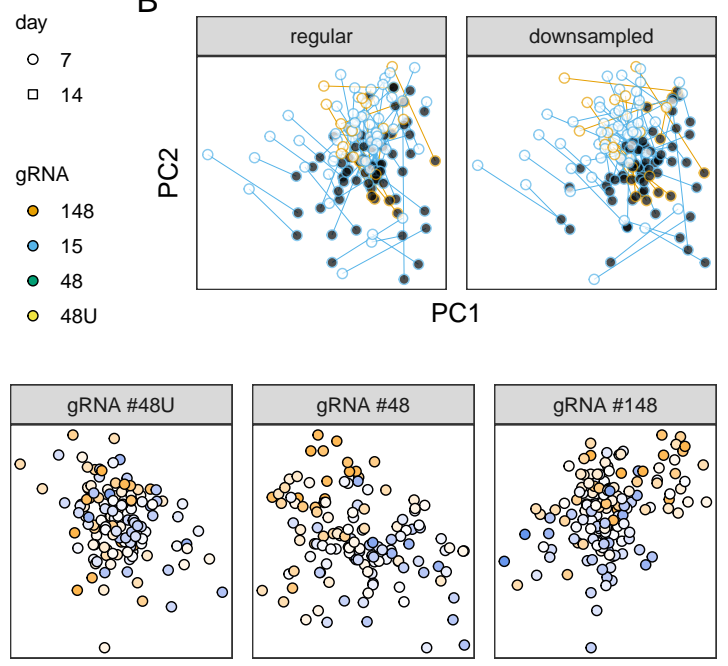

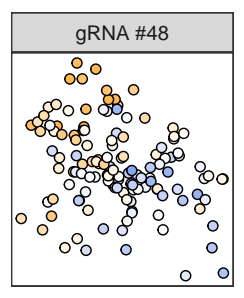

PC1
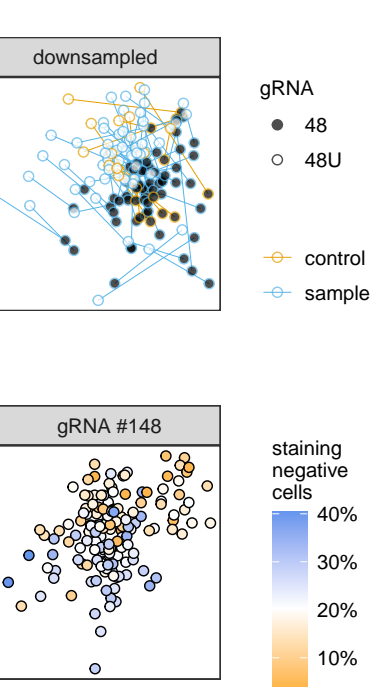

\%

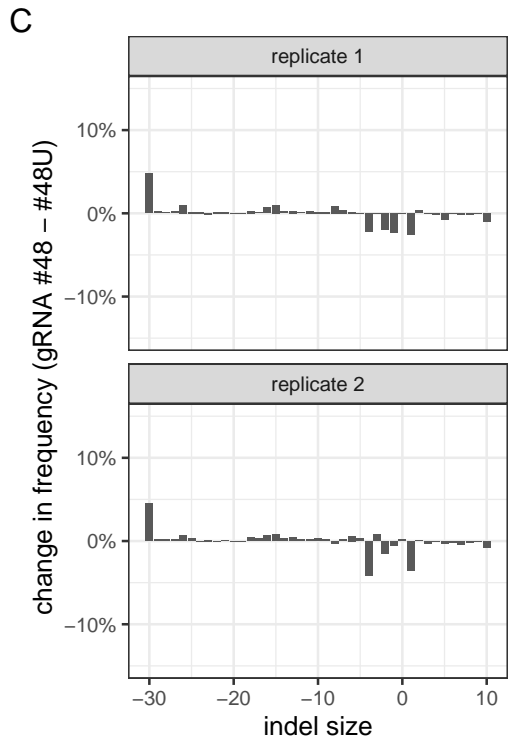

Fig. 4. Efficiency of mutagenesis affects DNA repair outcomes. (A) Relationship between flow cytometry samples. gRNA \#48U is a weaker version of \#48. $\mathrm{N}=4-6$ biologically independent replicates. (B). Relationship between clones based on their indel profiles, analogous to Fig.2B. Only non-significant clones are shown for clarity. Biological replicates $(\mathrm{N}=2)$ were averaged. (C) Difference in indel frequency between the regular gRNA \#48 and its less active counterpart \#48U. Same display conventions as in Fig.2. (D) Relationship between clones based on their indel profiles, analogous to Fig.2B. Each clone is colored by the frequency of mutagenesis assayed by flow cytometry on day 14 using gRNA \#48U, a proxy for Cas9 activity. For clarity, only non-significant clones are depicted.

out above. We found that gRNA \#148 induced two different, prominent $10 \mathrm{bp}$ deletions with $2 \mathrm{bp}$ microhomology, whose frequency changed divergently in Trexl deficient clones. One of them, the only notable large indel to increase in frequency upon Trexl depletion, involved a G-homopolymer. Another divergent indel, a 4 bp deletion with 2 bp microhomology induced by gRNA \#48, was promoted by deficiencies in Prkdc, Dclre1c, Trp53bpl and Polm (but not Ercc1), which otherwise decreased the frequency of 2-5 bp indels. We believe targeting additional loci to find more such apparently divergent outcomes could be useful to learn the rules governing DNA-sequence specific DSB-repair.

\section{Efficient mutagenesis leads to increase in size of} small indels. Cas9 has a number of properties that make it likely to interfere with the DSB repair process. Among others, Cas9 can recut the DNA immediately after a perfect repair, may cut both sister chromatids simultaneously, stays bound to DNA after introducing the cut and might possess exonuclease activity. If Cas9 interferes with DSB repair in any of these ways, then manipulating its concentration or activity could result in changed indel profiles. To investigate this issue, we have challenged the library with a low efficiency gRNA \#48U, whose target sequence is identical to \#48. Unlike \#48, \#48U's scaffold is expressed as two independent molecules, the crRNA (containing the target-matching sequence) and the tracrRNA. Significantly fewer control cells transfected with this weak gRNA lost PigA expression compared to the strong one (around 12\% vs 80\%, see Fig.S2). We speculate this is a consequence of reduced amount of "productive" gRNA.

To investigate the effect of mutagenic efficiency on repair outcomes, we first compared the results of the flow cyto- metric assay using gRNAs of different strengths. Samples transfected using the weak gRNA \#48U clustered away not only from \#15 samples, which track deletion frequency, but also from the combined cluster of strong exonic gRNAs \#48 and \#148 (Fig.4A). This difference was unlikely to be purely driven by the lower flow cytometry read-out with the weak gRNA, because the input for PCA-transformation was mean and standard deviation normalized, which should remove information about the relative magnitude of mutagenesis. Furthermore, \#48U samples collected on day 14 posttransfection were further away from the \#48 and \#148 cluster than samples collected on day 7, which is contrary to the expectation of the observed principal components capturing the magnitude of mutagenic efficiency. We conclude that mutagenic efficiency qualitatively affects the results of the flow cytometry assay.

To test whether mutagenic efficiency affected small indel profiles as well, we compared the sequencing results of gRNAs \#48 and \#48U. The central cluster of controls and non-affected gene-deficient clones was clearly split between the two gRNAs (Fig.4B, left). Since mutated alleles were sequenced much more shallowly in \#48U samples, which could potentially affect the results, we downsampled all read counts to the lowest common denominator (450 reads) and found that the effect persisted (Fig.4B, right). Indel profiles from combined control clones transfected with the strong gRNA had a higher frequency of larger deletions ( 5 bp deletions and larger), and correspondingly lower frequency of small indels, than clones transfected with the low efficiency gRNA (Fig.4C). This shift was reminiscent of one observed in NHEJ-deficient clones (such as Lig4 and Xrcc4) and could be interpreted as a relative increase in DNA resection and 
MMEJ-activity. The magnitude of the effect was small (no indel size changed in frequency by more than $5 \%$ percentage points), but reproducible between biological replicates.

There was considerable variability in the mutagenic efficiency among control clones in the flow cytometric assays (Fig.1B). We speculated that these differences in control and non-significant clones will correlate with differences between indel profiles. To ensure the highest dynamic range, we used day 14, gRNA \#48U flow cytometry samples as a gauge, since in this sample only about $19 \%$ of the control cells are mutagenized. We found that mutagenic efficiency in this sample measured by flow cytometry correlated with divergence in indel profiles, as evidenced by the separation of clones in principal component space (Fig.4D).

\section{Discussion}

We investigated the consequences of Cas9 mutagenesis in a panel of homogenous DNA damage repair deficient mouse embryonic stem cells. We found that the frequency of the complex lesions and large deletions ( $>260 \mathrm{bp}$ ) is increased by NHEJ deficiency and decreased by deficiencies in resection and MMEJ repair ( $N b n$ and Polq). This is the first, systematic, quantitative assessment of this effect in a wide range of deficient clones, using an assay specifically designed to capture them. Large deletion frequency correlated with the increase in extent of microhomology and size of small indels. These result are consistent with the described functions of the identified genes and imply a continuity of underlying repair processes across large genomic distances. They also underscore the potential mutagenic danger of NHEJ inhibition, a common strategy for increasing the frequency of templated repair.

Our results also imply a strategy for decreasing the frequency of complex lesions, namely inhibition of MMEJ or resection, in particular by targeting $\mathrm{Nbn}$. While global inhibition will decrease cellular viability and, in case of resection, genomewide repair fidelity (by preventing homologous recombination), a more targeted approach may be viable, like combining Cas9 enzyme with a resection-inhibiting moiety. Combining this strategy with prime editing could further reduce damage in rare cases when a DSB occurs. Another potential application of resection inhibition is to expedite the production of engineered cell clones by reducing the incidence of cryptic complex lesions (38). Moreover, repair outcomes in a resection-deficient context are much more predictable and more likely to lead to frame-disrupting 1-2 bp deletions and insertion. However, we note that the frame-shifting phenotype caused by $1 \mathrm{bp}$ lesions may also be more likely to be rescued by genetic compensation (54).

A number of deficiencies in auxiliary repair genes had locusselective effects. This observation is fully consistent with the well-described substrate-specificity of the repair genes involved, and the fact local target sequence shapes repair outcomes. Combining local nuclease-coupled manipulation of DNA repair machinery (e.g. 27) and indel profile prediction may be a viable strategy for obtaining the desired editing outcomes at a wide range of targets with minimal disruption to physiological DNA repair.

We were surprised to discover that Trexl deficiency had altered indel profiles. Trex1, discovered in 1969 and purified three decades later, has been studied extensively for its role in preventing autoimmunity caused by excess of ssDNA in the cytosol (55-59). Its in vitro exonuclease activity is fully compatible with a role in DSB repair but, to our knowledge, this is the first report linking the two. The underlying mechanism is unknown. Trex 1 could potentially act upon DSB directly, for example during $\mathrm{S}$ phase, when it is involved in resolution of dicentric chromosomes $(60,61)$. Alternatively, the observed effect could be a secondary consequence of ssDNA accumulation, perhaps related to the increase in mutagenic repair upon transfection of non-homologous DNA reported by Richardson et al. (62).

We have shown that increased efficiency of Cas9-mediated mutagenesis correlated with MMEJ-like shift towards larger indels. We have noticed a similar effect before, when comparing different modes of Cas9 delivery (63). Multiple mutually non-exclusive causes for this are possible. More efficient Cas9 complex can recut the DNA after perfect repair sooner (potentially leading to chromatin state dependent repair modulation), cut both sister chromatids simultaneously more often (confounding HR repair), stay bound to DNA after introducing the cut longer on the average (64, perhaps interfering with the assembly of repair machinery or causing replication fork stalling or collapse) and exert its potential exonuclease activity more intensely (in vitro: 65, 66), than Cas9 of lower efficiency. Finally, the difference in observed profiles could in part be a temporary consequence of slower MMEJ repair dynamics. As long as new DSBs are being introduced, there is an excess of alleles under repair by the MMEJ pathway compared to the faster NHEJ pathway. Alleles in the process of being repaired cannot be amplified and are thus depleted from the observed indel profile. It is not trivial to figure out in which direction this process would push the indel profile, and what the magnitude of this effect is in our assay. An experiment using inducible gRNAs or inducible Cas9 of different strength would clarify this issue. However, we believe it is unlikely that the difference we observed is entirely due to this, as the effect persist, when mutagenesis is nearing saturation (Fig.S2, gRNAs \#15, \#48 and \#148). Our results warrant further investigation and urge caution when using high concentrations of nucleases.

Despite deriving all our clones simultaneously from a pure, single cell cloned line, we observed a variability in mutagenic efficiency between control clones (e.g. 19-63\% on day 7 with gRNA\#48). The first round of subcloning likely removed most of the genetic variability, both genomic and related to individual lentiviral transductions (reverse transcription and APOBEC-mediated mutagenesis). Therefore, we speculate that differences in efficiency were due to a stochastic, mitotically heritable, epigenetic process acting on the Cas 9 transgene, possibly position effect variegation. Since varying intensity of DSB introduction has influence on indel profile measurements, this might have precluded us from observing more subtle changes brought about by DNA repair deficien- 
bioRxiv preprint doi: https://doi.org/10.1101/2020.08.05.216739; this version posted August 5, 2020. The copyright holder for this preprint (which was not certified by peer review) is the author/funder, who has granted bioRxiv a license to display the preprint in perpetuity. It is made available under aCC-BY-NC 4.0 International license.

cies. Variation in mutagenic efficiency between Cas 9 clones needs to be carefully consider as a potential confounder, when studying DNA damage repair.

Many genes in our library had no clones with statistically significant changes in indel profiles. Since their knock-out is only presumed based on the absence of small, framepreserving indels, we cannot claim it as evidence of no function. We note that some clones bona fide deficient for core end joining genes, such as Xlf\#1, Xrcc4\#3 and Polq\#1 exhibited a very mild or completely absent phenotype, while other clones with similar genotypes had very strong phenotypes. This, combined with the fact no significant effect was observed for genes with well-described functions in end joining, such as Parpl, Lig1, Lig3, Ctip and Paxx, implies genetic compensation might play a role.

\section{ACKNOWLEDGEMENTS}

We would like to thank Lara Urban for help with running the PEER package, which in our case yielded the same results as PCA. Kärt Tomberg, llias GeorgakopoulosSoares, Ozdemirhan Serçin1 and Michał Barski offered useful comments on the manuscript. The bioRxiv version of the manuscript was typeset using a slightly modified Henriques Lab LATEX template. This work is funded by Wellcome Trust grant no. 098051.

\section{AUTHOR CONTRIBUTIONS}

M.K. designed, executed, analyzed and interpreted the experiments and wrote the paper. A.B. supervised the project and contributed to writing of the manuscript. F.A. processed indel profile data and made useful comments on the manuscript.

\section{COMPETING INTERESTS}

The authors declare no competing interests.

\section{Bibliography}

1. Agnel Sfeir and Lorraine S Symington. Microhomology-Mediated End Joining: A Back-up Survival Mechanism or Dedicated Pathway? Trends in biochemical sciences, 40(11):701714, nov 2015. ISSN 0968-0004. doi: 10.1016/j.tibs.2015.08.006.

2. Hiroko Koike-Yusa, Yilong Li, E-Pien Tan, Martin Del Castillo Velasco-Herrera, and Kosuke Yusa. Genome-wide recessive genetic screening in mammalian cells with a lentivira CRISPR-guide RNA library. Nature biotechnology, 32(3):267-73, mar 2014. ISSN 15461696. doi: $10.1038 /$ nbt.2800.

3. Megan van Overbeek, Daniel Capurso, Matthew M Carter, Matthew S Thompson, Elizabeth Frias, Carsten Russ, John S. Reece-Hoyes, Christopher Nye, Scott Gradia, Bastien Vidal, Jiashun Zheng, Gregory R Hoffman, Christopher K Fuller, and Andrew P May. DNA Repair Profiling Reveals Nonrandom Outcomes at Cas9-Mediated Breaks. Molecular Cell, 63(4): 633-646, 2016. ISSN 10974164. doi: 10.1016/j.molcel.2016.06.037.

4. E. Pien Tan, Yilong Li, Martin Del Castillo Velasco-Herrera, Kosuke Yusa, and Allan Bradley. Off-target assessment of CRISPR-Cas9 guiding RNAs in human iPS and mouse ES cells. Genesis, 53(2):225-236, 2015. ISSN 1526968X. doi: 10.1002/dvg.22835.

5. Felicity Allen, Luca Crepaldi, Clara Alsinet, Alexander J. Strong, Vitalii Kleshchevnikov, Pietro De Angeli, Petra Páleníková, Anton Khodak, Vladimir Kiselev, Michael Kosicki, Andrew R. Bassett, Heather Harding, Yaron Galanty, Francisco Muñoz-Martínez, Emmanouil Metzakopian, Stephen P. Jackson, and Leopold Parts. Predicting the mutations generated by repair of Cas9-induced double-strand breaks. Nature Biotechnology, nov 2018. ISSN 1087-0156. doi: 10.1038/nbt.4317.

6. Max W. Shen, Mandana Arbab, Jonathan Y. Hsu, Daniel Worstell, Sannie J. Culbertson, Olga Krabbe, Christopher A. Cassa, David R. Liu, David K. Gifford, and Richard I. Sherwood. Predictable and precise template-free CRISPR editing of pathogenic variants. Nature, 563(7733):646-651, nov 2018. ISSN 0028-0836. doi: 10.1038/s41586-018-0686-x.

7. Wei Chen, Aaron McKenna, Jacob Schreiber, Maximilian Haeussler, Yi Yin, Vikram Agarwal, William Stafford Noble, and Jay Shendure. Massively parallel profiling and predictive modeling of the outcomes of CRISPR/Cas9-mediated double-strand break repair. Nucleic Acids Research, jun 2019. ISSN 0305-1048. doi: 10.1093/nar/gkz487.

8. Ryan T. Leenay, Amirali Aghazadeh, Joseph Hiatt, David Tse, Theodore L. Roth, Ryan Apathy, Eric Shifrut, Judd F. Hultquist, Nevan Krogan, Zhenqin Wu, Giana Cirolia, Hera Canaj, Manuel D. Leonetti, Alexander Marson, Andrew P. May, and James Zou. Large datase enables prediction of repair after CRISPR-Cas9 editing in primary T cells. Nature Biotechnology, 37(9):1034-1037, sep 2019. ISSN 1087-0156. doi: 10.1038/s41587-019-0203-2.

9. Brenda R. Lemos, Adam C. Kaplan, Ji Eun Bae, Alexander E. Ferrazzoli, James Kuo, Ranjith P. Anand, David P. Waterman, and James E. Haber. CRISPR/Cas9 cleavages in budding yeast reveal templated insertions and strand-specific insertion/deletion profiles. Proceedings of the National Academy of Sciences, page 201716855, 2018. ISSN 0027-8424. doi: 10.1073/pnas. 1716855115 .

10. Xin Shi, Jia Shou, Mohammadreza M. Mehryar, Jingwei Li, Leyang Wang, Mo Zhang, Haiyan Huang, Xiaofang Sun, and Qiang Wu. Cas9 has no exonuclease activity resulting in staggered cleavage with overhangs and predictable di- and tri-nucleotide CRISPR insertions without template donor. Cell Discovery, 5(1):53, dec 2019. ISSN 2056-5968. doi: 10.1038/s41421-019-0120-z.
11. S J Boulton and S P Jackson. Saccharomyces cerevisiae Ku70 potentiates illegitimate DNA double-strand break repair and serves as a barrier to error-prone DNA repair pathways. The EMBO journal, 15(18):5093-103, sep 1996. ISSN 0261-4189.

12. Zhuobin Liang, Sham Sunder, Sivakumar Nallasivam, and Thomas E. Wilson. Overhang polarity of chromosomal double-strand breaks impacts kinetics and fidelity of yeast nonhomologous end joining. Nucleic Acids Research, 44(6):2769-2781, 2016. ISSN 13624962. doi: 10.1093/nar/gkw013.

13. Minli Wang, Weizhong Wu, Wenqi Wu, Bustanur Rosidi, Lihua Zhang, Huichen Wang, and George lliakis. PARP-1 and Ku compete for repair of DNA double strand breaks by distinct NHEJ pathways. Nucleic acids research, 34(21):6170-82, 2006. ISSN 1362-4962. doi: 10.1093/nar/gkl840.

14. Amy Marie Yu and Mitch McVey. Synthesis-dependent microhomology-mediated end joining accounts for multiple types of repair junctions. Nucleic acids research, 38(17):5706-17, sep 2010. ISSN 1362-4962. doi: 10.1093/nar/gkq379.

15. Joost Schimmel, Hanneke Kool, Robin van Schendel, and Marcel Tijsterman. Mutational signatures of non-homologous and polymerase theta-mediated end-joining in embryonic stem cells. The EMBO Journal, page e201796948, 2017. ISSN 0261-4189. doi: 10.15252/ embj.201796948.

16. Maria Jasin and Rodney Rothstein. Repair of Strand Breaks by Homologous Recombination. Cold Spring Harbor Perspectives in Biology, 5 VN - re(11):1-19, 2013. ISSN 19430264. doi: 10.1101/cshperspect.a012740.

17. Theodore L. Roth, Cristina Puig-Saus, Ruby Yu, Eric Shifrut, Julia Carnevale, P. Jonathan Li, Joseph Hiatt, Justin Saco, Paige Krystofinski, Han Li, Victoria Tobin, David N. Nguyen, Michael R. Lee, Amy L. Putnam, Andrea L. Ferris, Jeff W. Chen, Jean-Nicolas Schickel, Laurence Pellerin, David Carmody, Gorka Alkorta-Aranburu, Daniela del Gaudio, Hiroyuki Matsumoto, Montse Morell, Ying Mao, Min Cho, Rolen M. Quadros, Channabasavaiah B. Gurumurthy, Baz Smith, Michael Haugwitz, Stephen H. Hughes, Jonathan S. Weissman, Kathrin Schumann, Jonathan H. Esensten, Andrew P. May, Alan Ashworth, Gary M. Kupfer, Siri Atma W. Greeley, Rosa Bacchetta, Eric Meffre, Maria Grazia Roncarolo, Neil Romberg, Kevan C. Herold, Antoni Ribas, Manuel D. Leonetti, and Alexander Marson. Reprogramming human $\mathrm{T}$ cell function and specificity with non-viral genome targeting. Nature, page 1, jul 2018. ISSN 0028-0836. doi: 10.1038/s41586-018-0326-5.

18. Renata Martin, Kazuya Ikeda, Nobuko Uchida, M. Kyle Cromer, Toshinobu Nishimura, Daniel P Dever, Joab Camarena, Rasmus Bak, Anders Lausten, Martin R Jakobsen, Volker Wiebking, Vittorio Sebastiano, Hiromitsu Nakauchi, and Matthew H Porteus. Selection-free, high frequency genome editing by homologous recombination of human pluripotent stem cells using Cas9 RNP and AAV6. bioRxiv, page 252163, jan 2018. doi: 10.1101/252163.

19. Takeshi Maruyama, Stephanie K. Dougan, Matthias C. Truttmann, Angelina M. Bilate, Jessica R. Ingram, and Hidde L. Ploegh. Increasing the efficiency of precise genome editing with CRISPR-Cas9 by inhibition of nonhomologous end joining. Nature Biotechnology, 33 (5):538-542, may 2015. ISSN 15461696. doi: 10.1038/nbt.3190.

20. Van Trung Chu, Timm Weber, Benedikt Wefers, Wolfgang Wurst, Sandrine Sander, Klaus Rajewsky, and Ralf Kühn. Increasing the efficiency of homology-directed repair for CRISPRCas9-induced precise gene editing in mammalian cells. Nature biotechnology, 33(5):543-8, may 2015. ISSN 1546-1696. doi: 10.1038/nbt.3198.

21. Stephan Riesenberg and Tomislav Maricic. Targeting repair pathways with small molecules increases precise genome editing in pluripotent stem cells. Nature communications, $9(1)$ : 2164, jun 2018. ISSN 2041-1723. doi: 10.1038/s41467-018-04609-7.

22. Stephan Riesenberg, Manjusha Chintalapati, Dominik Macak, Philipp Kanis, Tomislav Maricic, and Svante Pääbo. Simultaneous precise editing of multiple genes in human cells. Nucleic Acids Research, aug 2019. ISSN 0305-1048. doi: 10.1093/nar/gkz669.

23. Vertex ramps up CRISPR repair, Mar 2019. ISSN 1546-1696. [Online; accessed 10. May 2020].

24. Tarun S. Nambiar, Pierre Billon, Giacomo Diedenhofen, Samuel B. Hayward, Angelo Taglialatela, Kunheng Cai, Jen-Wei Huang, Giuseppe Leuzzi, Raquel Cuella-Martin, Andrew Palacios, Anuj Gupta, Dieter Egli, and Alberto Ciccia. Stimulation of CRISPR-mediated homology-directed repair by an engineered RAD18 variant. Nature Communications, 10(1): 3395, dec 2019. ISSN 2041-1723. doi: 10.1038/s41467-019-11105-z.

25. Bruna S. Paulsen, Pankaj K. Mandal, Richard L. Frock, Baris Boyraz, Rachita Yadav, Srigokul Upadhyayula, Paula Gutierrez-Martinez, Wataru Ebina, Anders Fasth, Tomas Kirchhausen, Michael E. Talkowski, Suneet Agarwal, Frederick W. Alt, and Derrick J. Rossi. Ectopic expression of RAD52 and dn53BP1 improves homology-directed repair during CRISPR-Cas9 genome editing. Nature Biomedical Engineering, 1(11):878-888, nov 2017. ISSN 2157-846X. doi: 10.1038/s41551-017-0145-2.

26. Jun Song, Dongshan Yang, Jie Xu, Tianqing Zhu, Y. Eugene Chen, and Jifeng Zhang. RS-1 enhances CRISPR/Cas9- and TALEN-mediated knock-in efficiency. Nature Communications, 7:1-7, 2016. ISSN 20411723. doi: 10.1038/ncomms10548.

27. Holly A. Rees, Wei-Hsi Yeh, and David R. Liu. Development of hRad51-Cas9 nickase fusions that mediate HDR without double-stranded breaks. Nature Communications, 10(1): 2212, dec 2019. ISSN 2041-1723. doi: 10.1038/s41467-019-09983-4.

28. Beeke Wienert, David N. Nguyen, Alexis Guenther, Sharon J. Feng, Melissa N. Locke, Stacia K. Wyman, Jiyung Shin, Katelynn R. Kazane, Georgia L. Gregory, Matthew A. M. Carter, Francis Wright, Bruce R. Conklin, Alex Marson, Chris D. Richardson, and Jacob E. Corn. Timed inhibition of CDC7 increases CRISPR-Cas9 mediated templated repair. Nature Communications, 11(1):2109, Apr 2020. ISSN 2041-1723. doi: 10.1038/s41467-020-15845-1.

29. Ha Youn Shin, Chaochen Wang, Hye Kyung Lee, Kyung Hyun Yoo, Xianke Zeng, Tyler Kuhns, Chul Min Yang, Teresa Mohr, Chengyu Liu, and Lothar Hennighausen. CRISPR/Cas9 targeting events cause complex deletions and insertions at 17 sites in the mouse genome. Nature Communications, 8(May):1-10, 2017. doi: 10.1038/ncomms15464.

30. Michael Kosicki, Kärt Tomberg, and Allan Bradley. Repair of double-strand breaks induced by CRISPR-Cas9 leads to large deletions and complex rearrangements. Nature Biotechnology, 36(8):765, jul 2018. ISSN 1087-0156. doi: 10.1038/nbt.4192.

31. Fatwa Adikusuma, Sandra Piltz, Mark A. Corbett, Michelle Turvey, Shaun R. McColl, Karla J. Helbig, Michael R. Beard, James Hughes, Richard T. Pomerantz, and Paul Q. Thomas. Large deletions induced by Cas9 cleavage. Nature, 560(7717):E8-E9, aug 2018. ISSN 0028-0836. doi: 10.1038/s41586-018-0380-z. 
bioRxiv preprint doi: https://doi.org/10.1101/2020.08.05.216739; this version posted August 5, 2020. The copyright holder for this preprint (which was not certified by peer review) is the author/funder, who has granted bioRxiv a license to display the preprint in perpetuity. It is made available under aCC-BY-NC 4.0 International license.

32. Christopher E. Nelson, Yaoying Wu, Matthew P. Gemberling, Matthew L. Oliver, Matthew A. Waller, Joel D. Bohning, Jacqueline N. Robinson-Hamm, Karen Bulaklak, Ruth M. Castellanos Rivera, Joel H. Collier, Aravind Asokan, and Charles A. Gersbach. Long-term evaluation of AAV-CRISPR genome editing for Duchenne muscular dystrophy. Nature Medicine, 25(3):427-432, mar 2019. ISSN 1078-8956. doi: 10.1038/s41591-019-0344-3.

33. Joffrey Mianné, Lauren Chessum, Saumya Kumar, Carlos Aguilar, Gemma Codner, Marie Hutchison, Andrew Parker, Ann-Marie Mallon, Sara Wells, Michelle M Simon, Lydia Teboul, Steve D M Brown, and Michael R Bowl. Correction of the auditory phenotype in C57BL/6N mice via CRISPR/Cas9-mediated homology directed repair. Genome medicine, 8(1):16, feb 2016. ISSN 1756-994X. doi: 10.1186/s13073-016-0273-4.

34. Jean-Baptiste Renaud, Charlotte Boix, Marine Charpentier, Anne De Cian, Julien Cochennec, Evelyne Duvernois-Berthet, Loïc Perrouault, Laurent Tesson, Joanne Edouard, Reynald Thinard, Yacine Cherifi, Séverine Menoret, Sandra Fontanière, Noémie de Crozé, Alexandre Fraichard, Frédéric Sohm, Ignacio Anegon, Jean-Paul Concordet, and Carine Giovannangeli. Improved Genome Editing Efficiency and Flexibility Using Modified Oligonucleotides with TALEN and CRISPR-Cas9 Nucleases. Cell Reports, 14(9):2263-2272, mar 2016. ISSN 22111247. doi: 10.1016/j.celrep.2016.02.018.

35. Marie-Christine Birling, Laurence Schaeffer, Philippe André, Loic Lindner, Damien Maréchal, Abdel Ayadi, Tania Sorg, Guillaume Pavlovic, and Yann Hérault. Efficient and rapid generation of large genomic variants in rats and mice using CRISMERE. Scientific Reports, 7(January):43331, mar 2017. ISSN 2045-2322. doi: 10.1038/srep43331.

36. Gemma F. Codner, Joffrey Mianné, Adam Caulder, Jorik Loeffler, Rachel Fell, Ruairidh King Alasdair J. Allan, Matthew Mackenzie, Fran J. Pike, Christopher V. McCabe, Skevoulla Christou, Sam Joynson, Marie Hutchison, Michelle E. Stewart, Saumya Kumar, Michelle M. Simon, Loranne Agius, Quentin M. Anstee, Kirill E. Volynski, Dimitri M. Kullmann, Sara Wells, and Lydia Teboul. Application of long single-stranded DNA donors in genome editing: generation and validation of mouse mutants. BMC Biology, 16(1):70, dec 2018. ISSN 1741-7007. doi: 10.1186/s12915-018-0530-7.

37. Grégoire Cullot, Julian Boutin, Jérôme Toutain, Florence Prat, Perrine Pennamen, Caroline Rooryck, Martin Teichmann, Emilie Rousseau, Isabelle Lamrissi-Garcia, Véronique Guyonnet-Duperat, Alice Bibeyran, Magalie Lalanne, Valérie Prouzet-Mauléon, Béatrice Turcq, Cécile Ged, Jean-Marc Blouin, Emmanuel Richard, Sandrine Dabernat, François Moreau-Gaudry, and Aurélie Bedel. CRISPR-Cas9 genome editing induces megabasescale chromosomal truncations. Nature Communications, 10(1):1136, dec 2019. ISSN 2041-1723. doi: 10.1038/s41467-019-09006-2.

38. Isabel Weisheit, Joseph Kroeger, Rainer Malik, Julien Klimmt, Dennis Crusius, Angelika Dannert, Martin Dichgans, and Dominik Paquet. Detection of deleterious on-target effects after HDR-mediated CRISPR editing. bioRxiv, page 2020.03.27.012104, mar 2020. doi: 10.1101/2020.03.27.012104.

39. Kazuya Ikeda, Nobuko Uchida, Toshinobu Nishimura, Joseph White, Renata M. Martin, Hiromitsu Nakauchi, Vittorio Sebastiano, Kenneth I. Weinberg, and Matthew H. Porteus. Efficient scarless genome editing in human pluripotent stem cells. Nature Methods, 15(12): 1045-1047, dec 2018. ISSN 15487105. doi: 10.1038/s41592-018-0212-y.

40. Emily Rayner, Mary-Anne Durin, Rachael Thomas, Daniela Moralli, Sean M. O'Cathail, Ian Tomlinson, Catherine M. Green, and Annabelle Lewis. CRISPR-Cas9 Causes Chromosomal Instability and Rearrangements in Cancer Cell Lines, Detectable by Cytogenetic Methods. The CRISPR Journal, 2(6):406-416, dec 2019. ISSN 2573-1599. doi: 10.1089/crispr.2019.0006.

41. Alexis C. Komor, Yongjoo B. Kim, Michael S. Packer, John A. Zuris, and David R. Liu. Programmable editing of a target base in genomic DNA without double-stranded DNA cleavage. Nature, 61(16):5985-91, 2016. ISSN 0028-0836. doi: 10.1038/nature17946.

42. Andrew V. Anzalone, Peyton B. Randolph, Jessie R. Davis, Alexander A. Sousa, Luke W. Koblan, Jonathan M. Levy, Peter J. Chen, Christopher Wilson, Gregory A. Newby, Aditya Raguram, and David R. Liu. Search-and-replace genome editing without double-strand breaks or donor DNA. Nature, pages 1-1, oct 2019. ISSN 0028-0836. doi: 10.1038/ s41586-019-1711-4.

43. Patrick D. Hsu, David A. Scott, Joshua A. Weinstein, F. Ann Ran, Silvana Konermann, Vineeta Agarwala, Yinqing Li, Eli J. Fine, Xuebing Wu, Ophir Shalem, Thomas J. Cradick, Luciano A. Marraffini, Gang Bao, and Feng Zhang. DNA targeting specificity of RNA-guided Cas9 nucleases. Nature Biotechnology, 31(9):827-832, sep 2013. ISSN 10870156. doi: $10.1038 /$ nbt.2647.

44. Ruslan Strogantsev, Felix Krueger, Kazuki Yamazawa, Hui Shi, Poppy Gould, Megan Goldman-roberts, Kirsten Mcewen, Bowen Sun, Roger Pedersen, and Anne C Fergusonsmith. Allele-specific binding of ZFP57 in the epigenetic regulation of imprinted and nonimprinted monoallelic expression. Genome Biology, pages 1-18, 2015. ISSN 1465-6906. doi: 10.1186/s13059-015-0672-7.

45. Kosuke Yusa, S Tamir Rashid, Helene Strick-Marchand, Ignacio Varela, Pei-Qi Liu, David E Paschon, Elena Miranda, Adriana Ordóñez, Nicholas R F Hannan, Foad J Rouhani, Sylvie Darche, Graeme Alexander, Stefan J Marciniak, Noemi Fusaki, Mamoru Hasegawa, Michael C Holmes, James P Di Santo, David a Lomas, Allan Bradley, and Ludovic Vallier. Targeted gene correction of $\alpha 1$-antitrypsin deficiency in induced pluripotent stem cells. $\mathrm{Na}$ ture, 478(7369):391-4, oct 2011. ISSN 1476-4687. doi: 10.1038/nature10424.

46. Kendell Clement, Holly Rees, Matthew C. Canver, Jason M. Gehrke, Rick Farouni, Jonathan Y. Hsu, Mitchel A. Cole, David R. Liu, J. Keith Joung, Daniel E. Bauer, and Luca Pinello. CRISPResso2 provides accurate and rapid genome editing sequence analysis, mar 2019. ISSN 15461696

47. Alexander Dobin, Carrie A. Davis, Felix Schlesinger, Jorg Drenkow, Chris Zaleski, Sonali Jha, Philippe Batut, Mark Chaisson, and Thomas R. Gingeras. STAR: Ultrafast universa RNA-seq aligner. Bioinformatics, 29(1):15-21, jan 2013. ISSN 13674803. doi: 10.1093/ bioinformatics/bts635.

48. Michael Kosicki. Cas9-induced on-target genomic damage, 2019.

49. Phu Van, Wenxin Jiang, Raphael Gottardo, and Greg Finak. ggCyto: next generation opensource visualization software for cytometry. Bioinformatics (Oxford, England), 34(22):39513953, 2018. ISSN 1367-4811. doi: 10.1093/bioinformatics/bty441.

50. Greg Finak, Jacob Frelinger, Wenxin Jiang, Evan W Newell, John Ramey, Mark M Davis, Spyros A Kalams, Stephen C De Rosa, and Raphael Gottardo. OpenCyto: an open source infrastructure for scalable, robust, reproducible, and automated, end-to-end flow cytometry data analysis. PLoS computational biology, 10(8):e1003806, aug 2014. ISSN 1553-7358. doi: 10.1371/journal.pcbi.1003806.

51. R Development Core Team. R: A language and environment for statistical computing. $R$ Foundation for Statistical Computing, Vienna, Austria. URL http://www.R-project.org/., 2013. ISSN 16000706.

52. Hadley Wickham, Mara Averick, Jennifer Bryan, Winston Chang, Lucy McGowan, Romain François, Garrett Grolemund, Alex Hayes, Lionel Henry, Jim Hester, Max Kuhn, Thomas Pedersen, Evan Miller, Stephan Bache, Kirill Müller, Jeroen Ooms, David Robinson, Dana Seidel, Vitalie Spinu, Kohske Takahashi, Davis Vaughan, Claus Wilke, Kara Woo, and Hiroaki Yutani. Welcome to the Tidyverse. Journal of Open Source Software, 4(43):1686, Nov 2019. ISSN 2475-9066. doi: 10.21105/joss.01686.

53. Claus O. Wilke. Fundamentals of Data Visualization: A Primer on Making Informative and Compelling Figures. O'Reilly Media, Apr 2019. ISBN 978-149203108-6.

54. Lukas Bartonek, Daniel Braun, and Bojan Zagrovic. Frameshifting preserves key physicochemical properties of proteins. Proceedings of the National Academy of Sciences, 117 (11):5907-5912, Mar 2020. ISSN 0027-8424. doi: 10.1073/pnas.1911203117.

55. T Lindahl, J A Gally, and G M Edelman. Properties of deoxyribonuclease 3 from mammalian tissues. The Journal of biological chemistry, 244(18):5014-9, sep 1969. ISSN 0021-9258.

56. Matthias Höss, Peter Robins, Thomas J.P. Naven, Darryl J.C. Pappin, John Sgouros, and Tomas Lindahl. A human DNA editing enzyme homologous to the Escherichia coli DnaQ/MutD protein. EMBO Journal, 1999. ISSN 02614189. doi: 10.1093/emboj/18.13.3868.

57. Dan J. Mazur and Fred W. Perrino. Identification and expression of the TREX1 and TREX2 cDNA sequences encoding mammalian 3'-5' exonucleases. Journal of Biological Chemistry, 274(28):19655-19660, jul 1999. ISSN 00219258. doi: 10.1074/jbc.274.28.19655.

58. Yanick J. Crow, Bruce E. Hayward, Rekha Parmar, Peter Robins, Andrea Leitch, Manir Ali, Deborah N. Black, Hans Van Bokhoven, Han G. Brunner, Ben C. Hamel, Peter C. Corry, Frances M. Cowan, Suzanne G. Frints, Joerg Klepper, John H. Livingston, Sally Ann Lynch, Roger F. Massey, Jean François Meritet, Jacques L. Michaud, Gerard Ponsot, Thomas Voit, Pierre Lebon, David T. Bonthron, Andrew P. Jackson, Deborah E. Barnes, and Tomas Lindahl. Mutations in the gene encoding the 3'-5' DNA exonuclease TREX1 cause AicardiGoutières syndrome at the AGS1 locus. Nature Genetics, 38(8):917-920, aug 2006. ISSN 10614036. doi: 10.1038/ng1845.

59. Daniel B. Stetson, Joan S. Ko, Thierry Heidmann, and Ruslan Medzhitov. Trex1 Prevents Cell-Intrinsic Initiation of Autoimmunity. Cell, 134(4):587-598, aug 2008. ISSN 00928674. doi: 10.1016/j.cell.2008.06.032.

60. John Maciejowski, Aikaterini Chatzipli, Alexandra Dananberg, Titia de Lange, and Peter Campbell. APOBEC3B-dependent kataegis and TREX1-driven chromothripsis in telomere crisis. bioRxiv, page 725366, aug 2019. doi: 10.1101/725366.

61. John Maciejowski, Yilong Li, Nazario Bosco, Peter J. Campbell, and Titia De Lange. Chromothripsis and Kataegis Induced by Telomere Crisis. Cell, 163(7):1641-1654, dec 2015. ISSN 10974172. doi: 10.1016/j.cell.2015.11.054.

62. C. D. Richardson, G. J. Ray, N. L. Bray, and J. E. Corn. Non-homologous DNA increases gene disruption efficiency by altering DNA repair outcomes. Nature Communications, 7: 1-7, 2016. ISSN 20411723. doi: 10.1038/ncomms 12463.

63. Michael Kosicki, Sandeep S. Rajan, Flaminia C. Lorenzetti, Hans H. Wandall, Yoshiki Narimatsu, Emmanouil Metzakopian, and Eric P. Bennett. Dynamics of Indel Profiles Induced by Various CRISPR/Cas9 Delivery Methods. Progress in Molecular Biology and Translational Science, 152:49-67, jan 2017. ISSN 1877-1173. doi: 10.1016/BS.PMBTS.2017.09.003.

64. Samuel H. Sternberg, Sy Redding, Martin Jinek, Eric C. Greene, and Jennifer A. Doudna. DNA interrogation by the CRISPR RNA-guided endonuclease Cas9. Nature, 507(7490): 62-67, mar 2014. ISSN 0028-0836. doi: 10.1038/nature13011.

65. Martin Jinek, Krzysztof Chylinski, Ines Fonfara, Michael Hauer, Jennifer A Doudna, and Emmanuelle Charpentier. A Programmable Dual-RNA - Guided DNA Endonuclease in Adaptice Bacterial Immunity. Science (New York, N.Y.), 337(August):816-822, aug 2012. ISSN 0036-8075. doi: 10.1126/science. 1225829.

66. Anthony A. Stephenson, Austin T. Raper, and Zucai Suo. Bidirectional Degradation of DNA Cleavage Products Catalyzed by CRISPR/Cas9. Journal of the American Chemical Society, 140(10):3743-3750, 2018. ISSN 15205126. doi: 10.1021/jacs.7b13050. 
bioRxiv preprint doi: https://doi.org/10.1101/2020.08.05.216739; this version posted August 5, 2020. The copyright holder for this preprint (which was not certified by peer review) is the author/funder, who has granted bioRxiv a license to display the preprint in perpetuity. It is made available under aCC-BY-NC 4.0 International license.
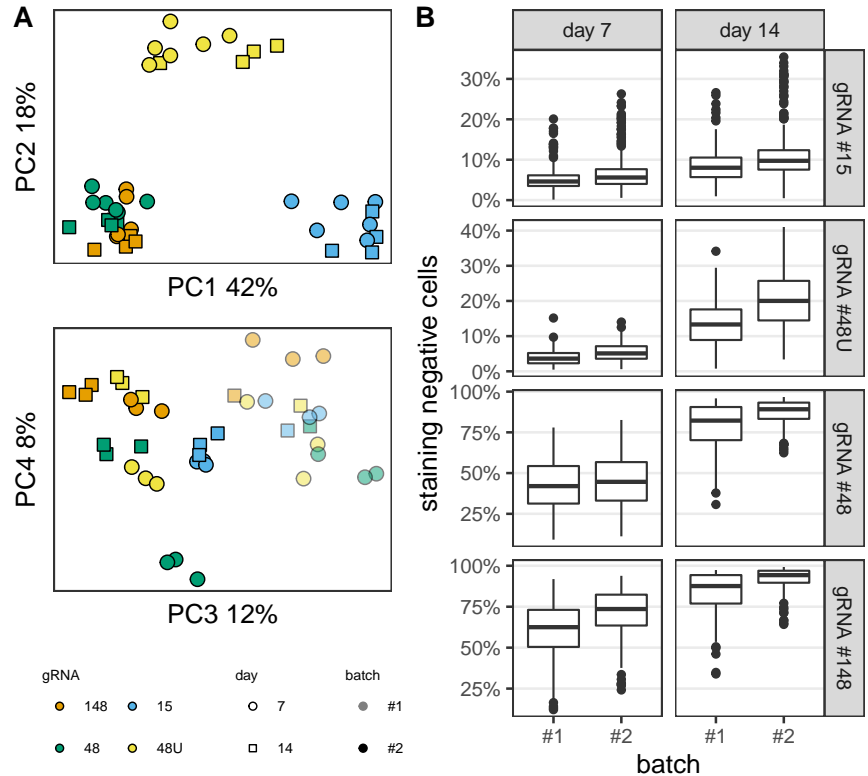

Fig. S1. Batch effect in the gene expression assay. (A) Relationships between samples based on surface gene expression. (B) Frequency of cells negative for the expression of target genes, broken down by batch. 

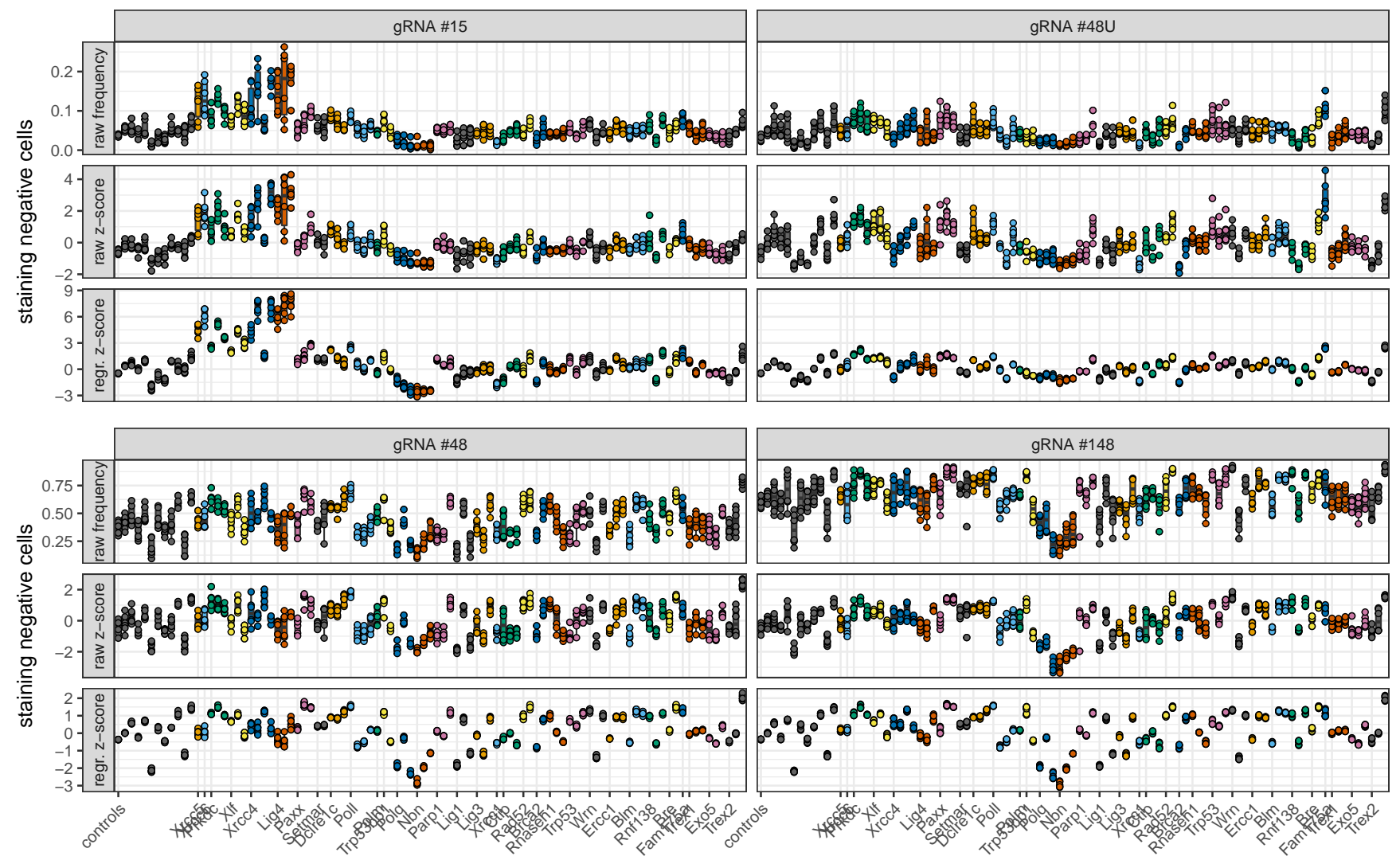

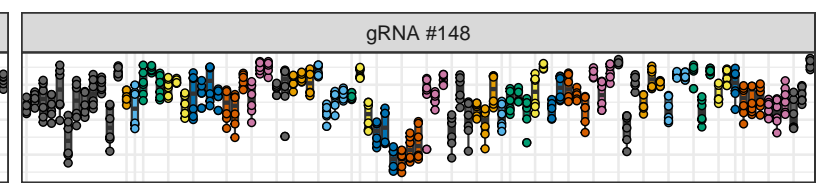

B
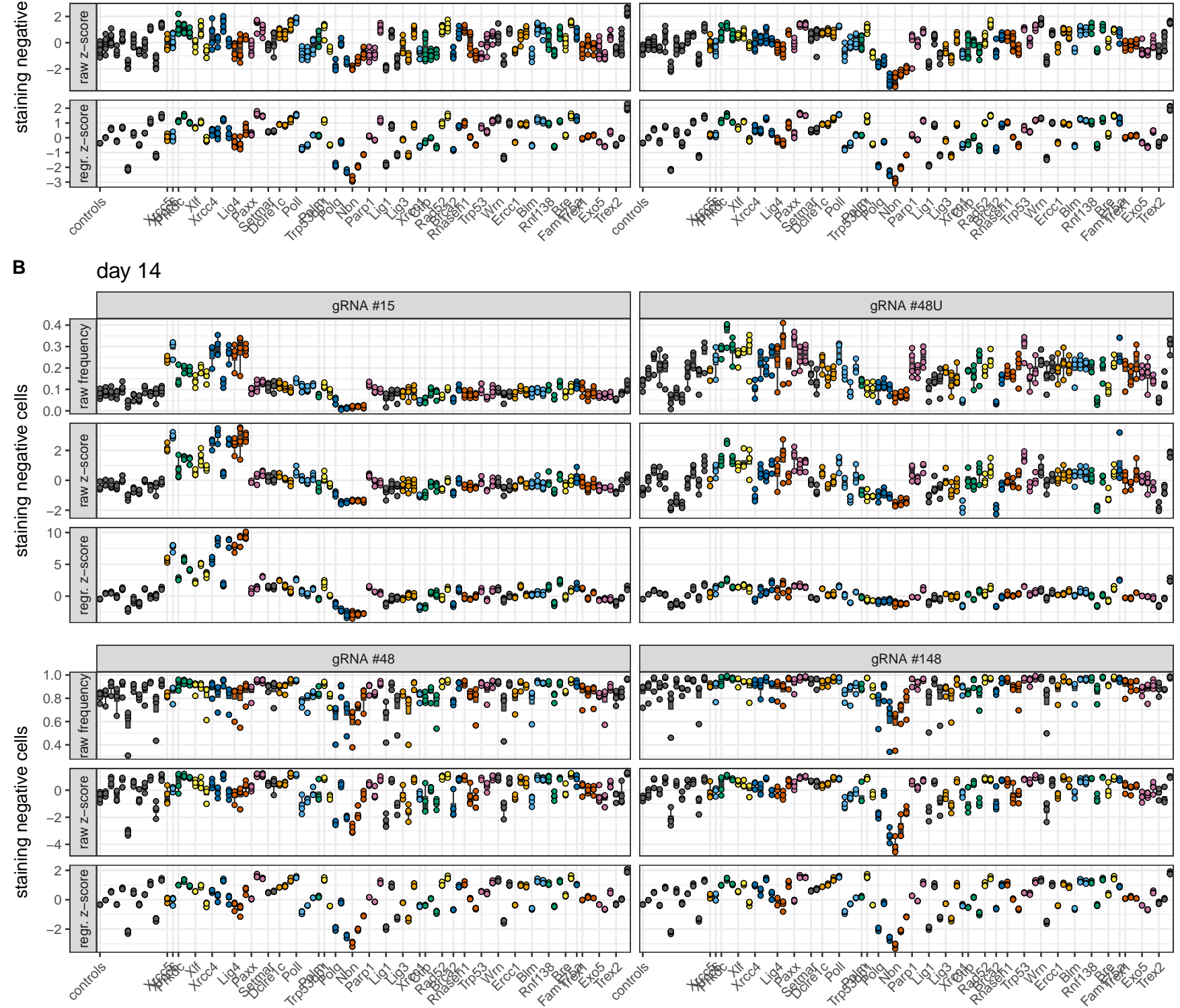

Fig. S2. Gene expression negative cells - raw frequency, raw z-score and PCA-regressed z-score. (A) Day 7 post-transfection. (B). Day 14. Same display conventions as in Fig.1B. 
bioRxiv preprint doi: https://doi.org/10.1101/2020.08.05.216739; this version posted August 5, 2020. The copyright holder for this preprint (which was not certified by peer review) is the author/funder, who has granted bioRxiv a license to display the preprint in perpetuity. It is made available under aCC-BY-NC 4.0 International license.
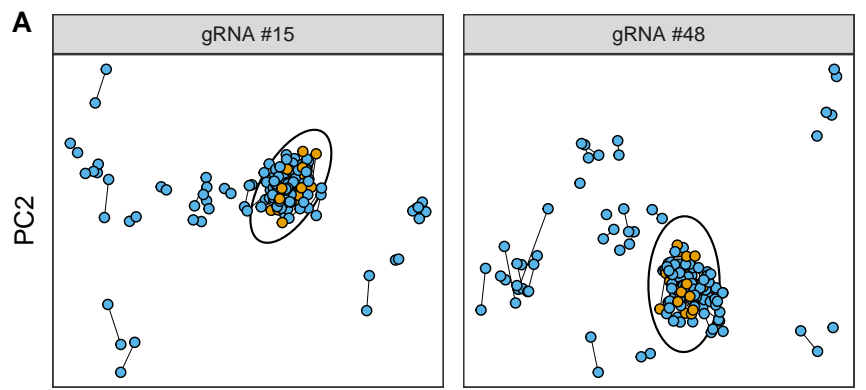

PC1
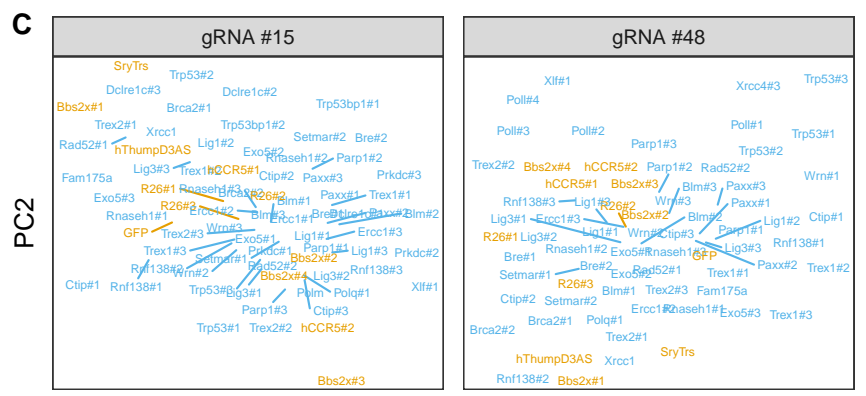

PC1
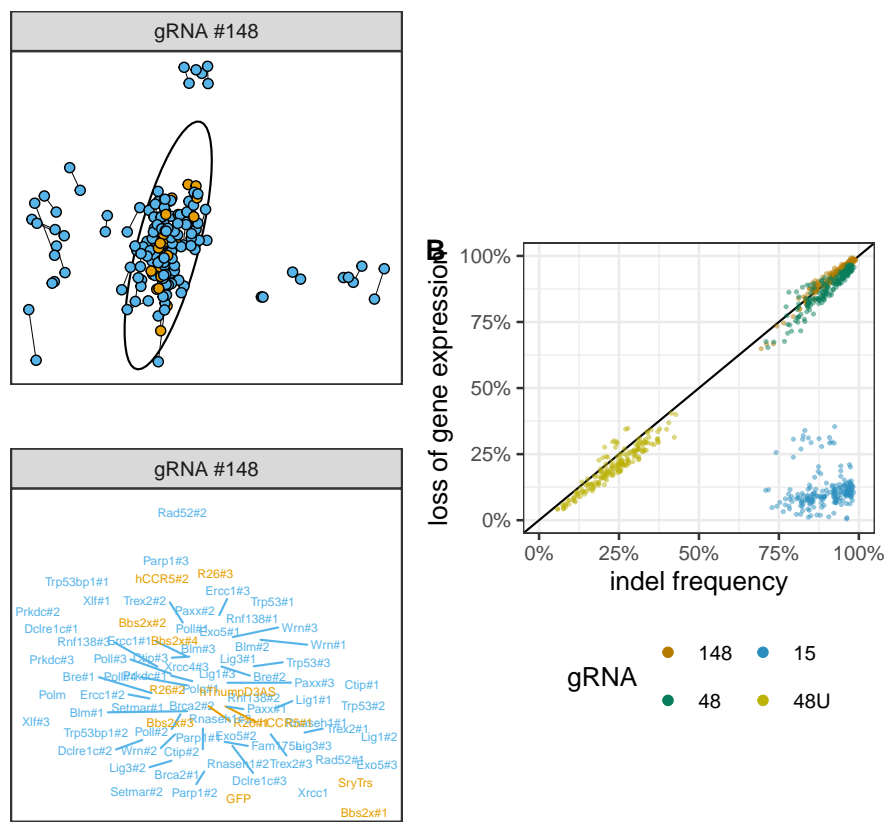

gRNA

- 148 - 15

- $48 \cdot 48 \mathrm{U}$

Fig. S3. Assay reproducibility and relationships between non-significant clones. (A) Relationships between replicate measurements of indel profiles. Ellipse is $99 \%$ confidence interval of a bivariate normal distribution fitted to control clones (orange points). (B) Comparison of the frequency of mutated alleles as measured by targeted sequencing of short-range PCR products and flow cytometric measurement of protein expression. NB: only large deletions and complex rearrangements, and not small indels, result in loss of gene expression with intronic gRNA \#15 (C) Relationships between clones not significantly different from controls, compare Fig.2B. Control clones are in orange. Biological replicates $(\mathrm{N}=2)$ were averaged. 
bioRxiv preprint doi: https://doi.org/10.1101/2020.08.05.216739; this version posted Auqust 5, 2020. The copyright holder for this preprint (which was not certified by peer review) is the author/funder, who has granted bioRxiv a license to display the preprint in perpetuity. It is made available under aCC-BY-NC 4.0 International license.
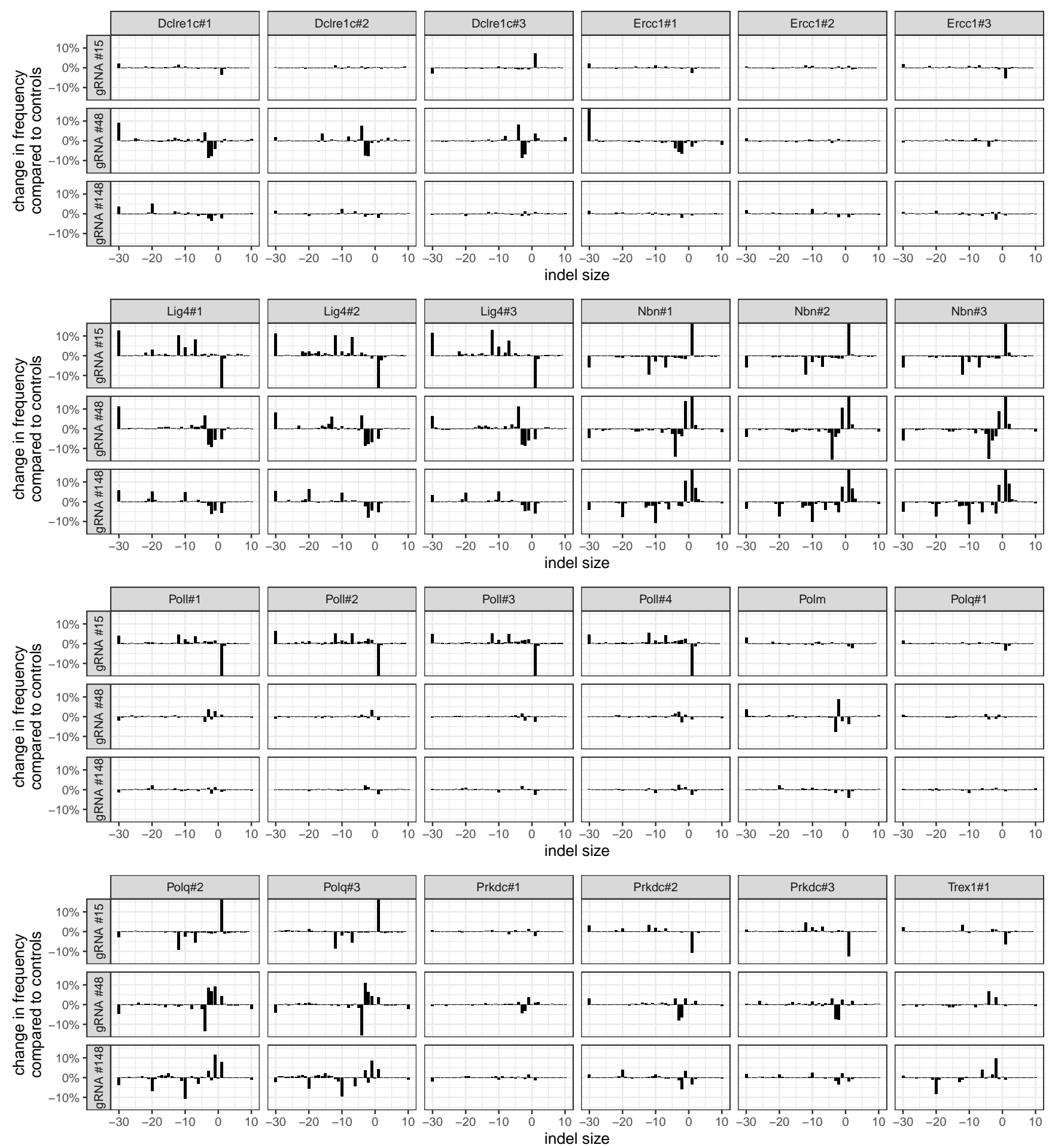
bioRxiv preprint doi: https://doi.org/10.1101/2020.08.05.216739; this version posted Auqust 5, 2020. The copyright holder for this preprint (which was not certified by peer review) is the author/funder, who has granted bioRxiv a license to display the preprint in perpetuity. It is made available under aCC-BY-NC 4.0 International license.
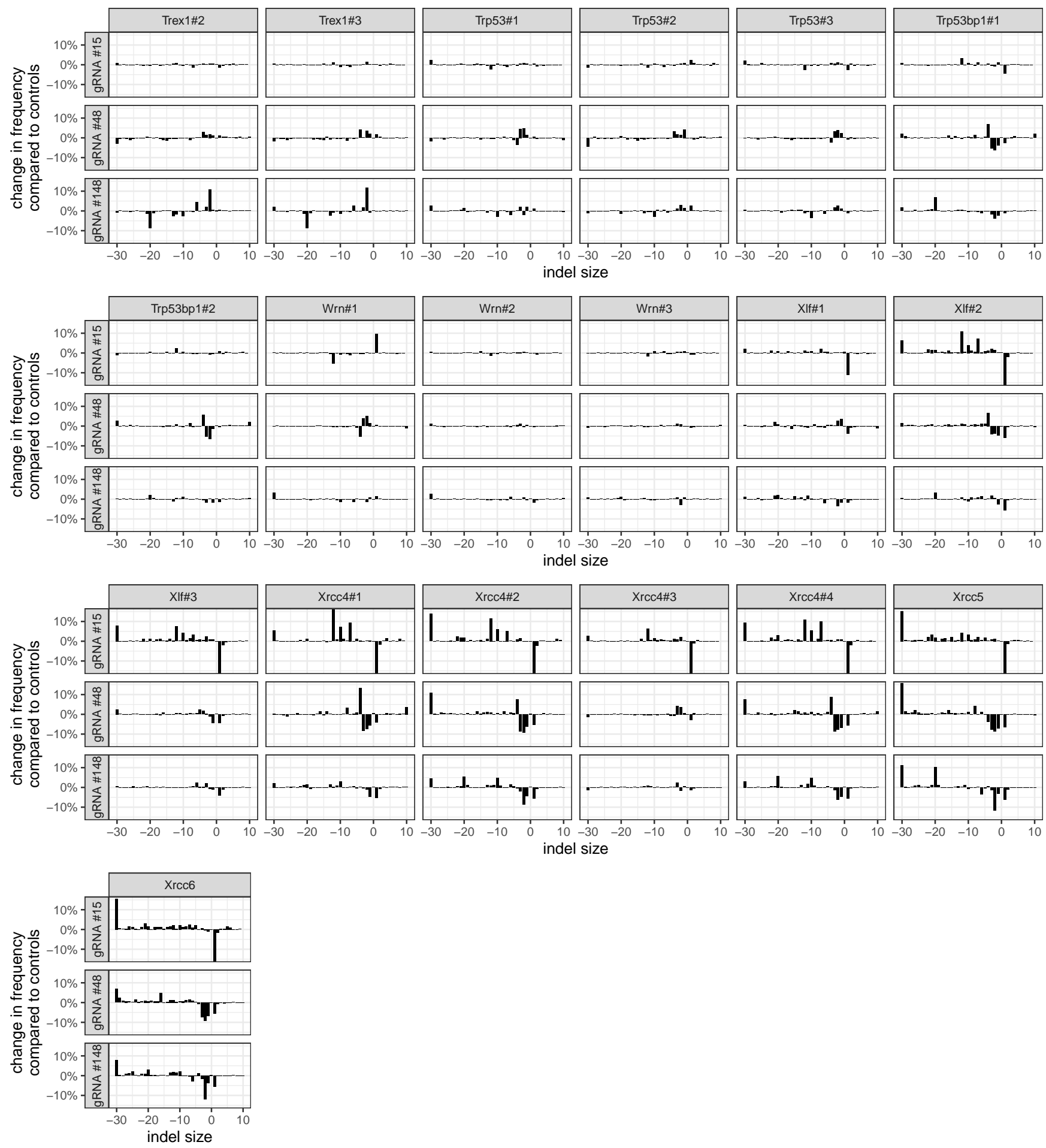

Fig. S4. Indel profiles of genes with at least one significant clone. Y-axis is truncated at $-15 \%$ and $+15 \%$ for clarity. Figure spans two pages. Same display conventions as in Fig.2. 